\title{
Exploring BODIPY Derivatives as Singlet Oxygen Photosensitizers for PDT $^{\dagger}$
}

\author{
Ruth Prieto-Montero ${ }^{1}$ (D), Alejandro Prieto-Castañeda ${ }^{2}$ (D), Rebeca Sola-Llano ${ }^{1}$ (iD, \\ Antonia R. Agarrabeitia ${ }^{2}$ (D), David García-Fresnadillo² (D), Iñigo López-Arbeloa ${ }^{1}$ (D), \\ Angeles Villanueva ${ }^{3,4}$ (D), María J. Ortiz ${ }^{2 *}$ (D), Santiago de la Moya ${ }^{2 *}$ (D) and \\ Virginia Martínez-Martínez ${ }^{1}$ * iD \\ ${ }^{1}$ Departamento de Química Física, Facultad de Ciencia y Tecnología, Universidad del País Vasco, Bilbao, Spain \\ ${ }^{2}$ Departamento de Química Orgánica, Facultad de CC. Químicas, Universidad Complutense de Madrid, Madrid, Spain \\ ${ }^{3}$ Departamento de Biología, Universidad Autónoma de Madrid, Madrid, Spain \\ ${ }^{4}$ IMDEA Nanociencia, Madrid, Spain
}

Received 30 September 2019, accepted 20 December 2019, DOI: 10.1111/php.13232

\begin{abstract}
This minireview is devoted to honoring the memory of Dr. Thomas Dougherty, a pioneer of modern photodynamic therapy (PDT). It compiles the most important inputs made by our research group since 2012 in the development of new photosensitizers based on BODIPY chromophore which, thanks to the rich BODIPY chemistry, allows a finely tuned design of the photophysical properties of this family of dyes to serve as efficient photosensitizers for the generation of singlet oxygen. These two factors, photophysical tuning and workable chemistry, have turned BODIPY chromophore as one of the most promising dyes for the development of improved photosensitizers for PDT. In this line, this minireview is mainly related to the establishment of chemical methods and structural designs for enabling efficient singlet oxygen generation in BODIPYs. The approaches include the incorporation of heavy atoms, such as halogens (iodine or bromine) in different number and positions on the BODIPY scaffold, and also transition metal atoms, by their complexation with $\operatorname{Ir}$ (III) center, for instance. On the other hand, lowtoxicity approaches, without involving heavy metals, have been developed by preparing several orthogonal BODIPY dimers with different substitution patterns. The advantages and drawbacks of all these diverse molecular designs based on BODIPY structural framework are described.
\end{abstract}

\section{INTRODUCTION}

Developing photosensitizers (PSs) for the generation of reactive oxygen species (ROS), including singlet molecular oxygen $\left({ }^{1} \mathrm{O}_{2}\right)$, is an active research field. These species result useful for different applications, such as photoswitchable wastewater treatment, fine chemical synthesis (e.g. selective oxidations), sterilization (including ${ }^{1} \mathrm{O}_{2}$ blood sterilization), sunlight-activated agricultural treatments against different plagues (weeds, microbes, insects,

*Corresponding authors emails: virginia.martinez@ehu.eus (Virginia MartínezMartínez),santmoya@ucm.es (Santiago de la Moya),mjortiz@quim.ucm.es (María J. Ortiz)

${ }^{\dagger}$ This article is part of a Special Issue dedicated to Dr. Thomas Dougherty (C) 2020 American Society for Photobiology fungi, etc.) or PDT (1-3). PDT is based on the selective destruction of pathological cells (acne, cancer tumor, microorganisms, etc.) by the action of cytotoxic ROS, mainly ${ }^{1} \mathrm{O}_{2}$, locally generated by the combined action of light and a PS. In this context, the PS should be selectively localized in the specific tissue region limiting undesired effects on healthy tissues (4-7). Therefore, PDT, and mainly cancer PDT, must be highlighted, due to its minimally invasive character, offering important advantages in comparison with traditional treatments.

Although a plethora of chemical systems (molecular and polymeric) have been described as successful PSs for ${ }^{1} \mathrm{O}_{2}$ generation, advanced PSs for clinical PDT are necessary in order to overcome the limitations of the current (recently proposed or even marketed) PSs for PDT (7). In this sense, dark toxicity, low absorption coefficients, particularly into the therapeutic spectral window $(650-875 \mathrm{~nm})$, low photostability, low selectivity and low biocompatibility are the most important shortcomings. Moreover, photoactive systems able to exhibit not only efficient ${ }^{1} \mathrm{O}_{2}$ generation under light irradiation but also fluorescence (a balanced dual activity of competitive photophysical pathways) are of special interest to advance in future specific theragnostic applications based on PDT treatment and diagnosis by fluorescence bioimaging (PDT + bioimaging). Such challenging objectives have prompted scientific efforts in this research area during the last years. In this context, the BODIPY (4,4-difluoro-4-bora3a,4a-diaza-s-indacene) scaffold has been revealed as an interesting chemical structure to develop PSs for ${ }^{1} \mathrm{O}_{2}$ generation (8) with improved features for clinical application, including PDT (9-12) and theragnosis (13). This article compiles the most important results reported by our research group in this line since 2012, showing representative examples of how the chemical manipulation of the BODIPY core allows the tuning of its photophysical behavior offering a wide range of possibilities for PDT. To cite some examples, here we describe several heavy-atom-based PSs such as halogenated BODIPYs with a different number of iodine or bromine atoms and different substitution patterns, or various BODIPYs chelated to a transition metal center, as well as several orthogonal BODIPY dimers as heavy-atom-free PSs. We also show the most relevant results from photodynamic experiments "in vitro" performed with different BODIPY photosensitizers and 
HeLa cells under similar conditions, which allowed elucitating the best BODIPY-based PS candidates, highlighting the advantages and disadvantages found in each series of compounds and, therefore, in their conceptual approach.

Furthermore, in order to understand the key factors involved in the design of such BODIPY-based PSs, a general overview about the most important aspects controlling the production of ${ }^{1} \mathrm{O}_{2}$ by photosensitization, as well as about the drawbacks (from synthetic, photophysical or biomedical viewpoints) of the current, most important PSs for ${ }^{1} \mathrm{O}_{2}$ generation, is also shown.

\section{Singlet oxygen production by photosensitization}

Generally, a photosensitized reaction is defined as the absorption of light by a PS, which induces a modification of another molecular species $(14,15)$. Particularly, the generation of ROS via photosensitization processes is mainly based on the nonradiative deactivation of the first triplet excited state $\left(\mathrm{T}_{1}\right)$ of the PS by two possible mechanisms (Fig. 1): (1) the Type I mechanism, where the deactivation involves electron transfer from the excited PS to the ground state of molecular oxygen, ${ }^{3} \mathrm{O}_{2}\left({ }^{3} \Sigma_{\mathrm{g}}\right)$, in order to generate different reactive oxygen species $\left(\mathrm{O}_{2}{ }^{\cdot-}, \mathrm{HO}^{\circ}\right.$, etc.) (16) and (2) the Type II mechanism, where the excitation energy is transferred, by a collisional process, to molecular oxygen, exciting it to its lowest $\mathrm{S}_{1}$ state ${ }^{1} \mathrm{O}_{2}\left({ }^{1} \Delta_{\mathrm{g}}\right)$, namely singlet molecular oxygen. The latter mechanism is usually considered as the main route in PDT (1-3); therefore, a more detailed knowledge of Type II mechanism, and the key requirements to control it, is necessary to advance in the design of new PSs for PDT.

In order to produce ${ }^{1} \mathrm{O}_{2}$ efficiently, the $\mathrm{S}_{1}$ excited state of the PS must display an efficient intersystem crossing (ISC) process to populate the triplet excited state $\left(\mathrm{T}_{1}\right)$. Then, $\mathrm{T}_{1}$ state undergoes de-excitation coupled to the excitation of a molecule of oxygen to its singlet state by Dexter energy transfer mechanism (Fig. 1). Since ISC is a spin-forbidden transition, it becomes more probable when electron spin-orbit coupling is non-negligible. The spin-orbit coupling consists of the interaction of the electron magnetic moments associated with the spin $(s)$ and the orbital angular momentum $(l)$, respectively. One consequence of electron spin-orbit coupling is the mixing of states of different multiplicity (e.g. $\mathrm{S}_{1}$ with $\mathrm{T}_{\mathrm{n}}$ ). As a result, the involved triplet $\mathrm{T}_{\mathrm{n}}$ state takes on some singlet-state character, and vice versa, the $S_{1}$ takes on some triplet character $(17,18)$. Owing to this quantum mixing, the forbidden $\mathrm{S}_{1} \rightarrow \mathrm{T}_{\mathrm{n}}$ transition can take place (ISC), finally populating $\mathrm{T}_{1}$ state. Then, by a collisional energy transfer process, the molecular oxygen $\left({ }^{3} \mathrm{O}_{2}\right)$ is excited to its singlet state $\left({ }^{1} \mathrm{O}_{2}\right)$ (Fig. 1) (1-3). Therefore, electronic spin-orbit coupling is the key to enhance the production of ${ }^{1} \mathrm{O}_{2}$. This coupling can be promoted by the so-called heavy-atom effect, which corresponds to the enhancement of the rate of a spin-forbidden process by the presence of an atom of high atomic number that can be either part of the molecular structure (inner spin-orbit effect) or be present in the medium (external spin-orbit effect), but always close enough to the excited molecular entity. It has been demonstrated that, for efficient ${ }^{1} \mathrm{O}_{2}$ production by the Type II mechanism (Fig. 1), PS should fulfill the following conditions: high absorption coefficient in the spectral region of the excitation light, high ISC quantum yield, long triplet lifetime and triplet energy higher than the energy gap $\left(94.2 \mathrm{~kJ} \mathrm{~mol}^{-1}\right)$ existing between the singlet excited state $\left({ }^{1} \Delta_{\mathrm{g}}\right)$ and the ground state $\left({ }^{3} \Sigma_{\mathrm{g}}\right)$ of molecular oxygen.

The capability of a PS for generating ${ }^{1} \mathrm{O}_{2}$ is measured by its singlet oxygen generation quantum yield, $\Phi_{\Delta}$. Photosensitizers usually produce ${ }^{1} \mathrm{O}_{2}$ only from their excited triplet state, due to highly efficient ISC $\left(\Phi_{\text {ISC }} \sim 1\right)$ and concomitant spin-allowed energy transfer from such a triplet state to the ground state of molecular oxygen $\left(\mathrm{T}_{0}\right)$. These PSs can exhibit a maximum $\Phi_{\Delta}$ value of 1 (e.g. phenalenone in Fig. 2). On the other hand, there are special cases in which ${ }^{1} \mathrm{O}_{2}$ can be produced from the singlet state of the PS, leading to a theoretical maximum of $\Phi_{\Delta}=2$ $(1,14)$. However, this last mechanism is possible for a relatively small group of photosensitizers whose energy gap between the excited singlet and triplet state is higher than the energy gap between singlet oxygen and ground-state oxygen.

Finally, once ${ }^{1} \mathrm{O}_{2}$ is formed, several pathways can deactivate it: (1) luminescence emission (phosphorescence at ca. $1270 \mathrm{~nm}$ ), (2) chemical reaction with a substrate and (3) physical quenching (e.g. collisions with solvent and/or other quencher molecules) as a consequence of the long lifetime of ${ }^{1} \mathrm{O}_{2}\left(\tau_{\Delta}=1 / k_{\mathrm{d}}, k_{\mathrm{d}}\right.$ being the rate constant of ${ }^{1} \mathrm{O}_{2}$ deactivation by the solvent), often in the $\mu$ s range, although strongly dependent on the presence of the $\mathrm{O}-$ $\mathrm{H}$ and $\mathrm{C}-\mathrm{H}$ oscillators in the solvent molecules (around $3 \mu \mathrm{s}$ in water, $10 \mu \mathrm{s}$ in methanol, ca. $85 \mu \mathrm{s}$ in acetonitrile (ACN) or ca. $265 \mu$ s in chloroform).

\section{Photosensitizers for singlet oxygen generation}

As cited above, a high absorption coefficient of light in the spectral region used for the photoexcitation, a triplet state of proper

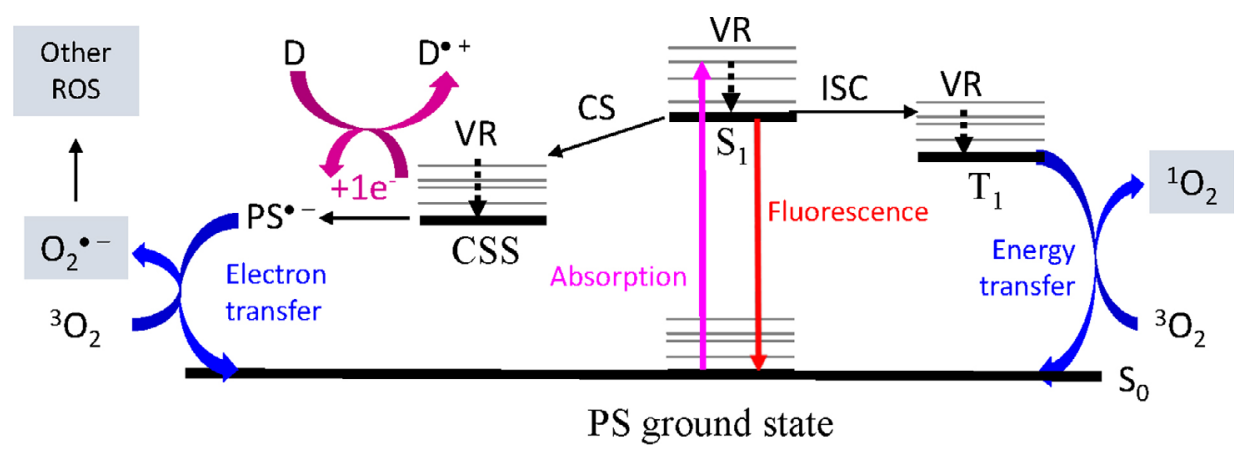

Figure 1. Jablonski diagram showing Type I and Type II mechanisms for photoinduced production of ROS involved in PDT. PS, photosensitizer; S, singlet excited state; $\mathrm{T}_{1}$, triplet excited state; CSS, charge-separated state; VR, vibrational relaxation; ISC, intersystem crossing; CS, charge separation; $\mathrm{D}$, electron donor. 
<smiles>O=C(O)c1c(Cl)c(Cl)c(Cl)c(Cl)c1C1=C2C=C(I)C(=O)C(I)C2Oc2c1cc(I)c(O)c2I</smiles>

Xanthene dyes<smiles>CN(C)c1ccc2nc3ccc(=[N+](C)C)cc-3sc2c1</smiles>

Phenothiazinium dyes

Methylene Blue<smiles>O=C1C=Cc2cccc3cccc1c23</smiles>

Aromatic hydrocarbons

Phenalenone

Rose Bengal<smiles>[R]c1c2ccc3[nH]c(ccc4[nH][nH]c5ccc([nH]5)c([R])c4ccc1n2)c3[R]</smiles>

Porphyrin<smiles>[R]c1c2nc(c([R])c3ccc([nH]3)c([R])c3nc(c([R])c4ccc1[nH]4)CC3)C=C2</smiles>

Chlorin<smiles>[R]c1c2nc(c([R])c3ccc([nH]3)c([R])c3nc(c([R])c4ccc1[nH]4)CC3)CC2</smiles>

Bacteriochlorin

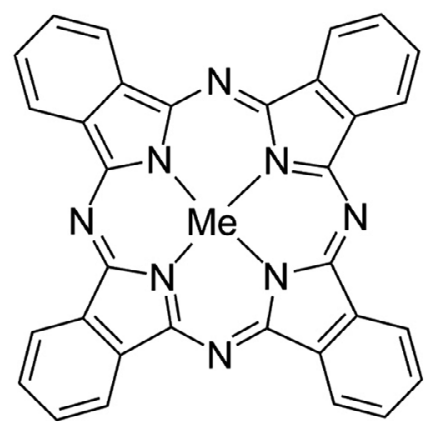

Metallophthalocyanine

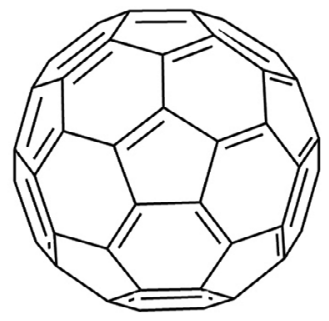

$\left[\mathrm{C}_{60}\right]$ Fullerene

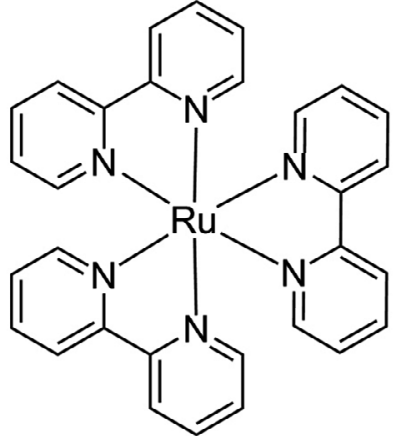

$\left[\mathrm{Ru}(\mathrm{bpy})_{3}\right]^{2+}$

Figure 2. Common PSs for ${ }^{1} \mathrm{O}_{2}$ generation.

energy $\left(E_{\mathrm{T}}>94 \mathrm{~kJ} \mathrm{~mol}^{-1}\right)$, a high quantum yield of triplet-state population $\left(\Phi_{\mathrm{T}}>0.4\right)$, a long triplet-state lifetime $\left(\tau_{\mathrm{T}}>1 \mu \mathrm{s}\right)$ and a high photostability are essential requisites for suitable PSs (14). In this regard, several molecular structures fulfill the condition of enhancing the population of their corresponding triplet excited states. The most representative PSs for singlet oxygen generation are described below.

Among the most commonly used PSs, rose bengal (RB), methylene blue $(\mathrm{MB})$ and phenalenone $(\mathrm{PN})$ must be highlighted as standard references to determine $\Phi_{\Delta}$ values for other PSs. RB (Fig. 2) is a xanthene-based dye with strong absorption bands in the visible (Vis) region and high efficiency for ${ }^{1} \mathrm{O}_{2}$ generation under irradiation in air-equilibrated polar solvents (e.g. $\lambda_{\mathrm{abs}}$
$550 \mathrm{~nm}, \Phi_{\Delta}=0.5-0.8$ in $\mathrm{ACN}$ or $\mathrm{MeOH}$, respectively). These factors make RB the most popular reference to quantify $\Phi_{\Delta}$ for Vis-absorbing PSs but only in polar media $(1,3)$. MB, a phenothiazine-based dye, with a strong absorbance into the therapeutic window (ca. $650 \mathrm{~nm}$ ) and significant $\Phi_{\Delta}$ (around 0.50 in polar solvents, e.g. methanol, ethanol and water), has been also used for such a purpose $(1,3)$. On the other hand, PN $\left(\lambda_{\mathrm{abs}} 360 \mathrm{~nm}\right.$, $\Phi_{\Delta}$ ca. 1) is considered as the universal reference photosensitizer for quantifying $\Phi_{\Delta}$ in both nonpolar and polar solvents, due to its high solubility in many different solvents of a broad polarity range (even in aqueous media by the $1 H$-phenalen-1-one-2-sulfonic acid derivative), with $\Phi_{\Delta}$ values near 1 independently of the solvent. However, the main shortcoming of $\mathrm{PN}$ is related to 
its limited absorption of light in the Vis region (absorption below $450 \mathrm{~nm}$ ) (19). Recently, a BODIPY derivative (3,5-dimethyl-2,6diiodo-8-(methylthio)BODIPY) has been postulated as an alternative standard photosensitizer for singlet oxygen production, under excitation either in the UV or in the visible range (20).

On the other hand, best PS candidates for PDT treatments must accomplish some additional criteria: (1) low cytotoxicity in the absence of light (dark toxicity); (2) selective accumulation in the pathological tissue; (3) limited in vivo stability in order to favor its removal after the PDT treatment; (4) high light absorption in the therapeutical window (spectral region of maximum light penetration into tissues; $\lambda_{\mathrm{abs}}>650 \mathrm{~nm}, \varepsilon \geq 5 \times$ $10^{4} \mathrm{M}^{-1} \mathrm{~cm}^{-1}$ ), to maximize the depth of light penetration into the irradiated tissue; (5) solubility in aqueous media; and (6) high ability to penetrate the cell membranes (4-7).

Regarding PSs for clinical PDT, porphyrins have been described as successful PDT PSs, (21-25), such as porfimer sodium (Photofrin ${ }^{\circledR}$; Fig. 3) (26,27), a hematoporphyrin-based PS consisting of a mixture of oligomeric polyporphyrins, linked together by ester- and ether-based chains, which has been approved for clinical use in the treatment of certain types of cancer. Photofrin ${ }^{\circledR}$ induces phototoxicity through a cellular uptake mechanism, relying on the intracellular oxygen levels. However, it exhibits low absorbance into the therapeutic window $(>650 \mathrm{~nm}$ ) and requires extended irradiation with high-energy sources. Moreover, both chemical complexity and high photobleaching rate decrease its PDT efficiency. Trying to overcome these drawbacks, a second generation of PDT PSs with improved features was developed (22-25).

The second generation of PDT PSs is confirmed by 5 -aminolevulinic acid (ALA, Levulan $\left.{ }^{\circledR}\right)(26-28)$ and several ALA derivatives (21), as well as some chlorin (a dihydroporphyrin) and bacteriochlorin (a tetrahydroporphyrin) derivatives (29,30). Note here that ALA is not a PDT PS itself, but a precursor of the PS known as protoporphyrin IX (PpIX in Fig. 3). ALA has received considerable interest in the treatment of shallow carcinomas. However, its therapeutic action is very superficial due to its poor penetration into the tissue (penetration depth ca. $1 \mathrm{~mm}$ ), which is an important drawback, which limits its clinical use. This problem has been partially addressed through the development of advanced ALA derivatives based on ALA ester, such as Metvix ${ }^{\circledR}$, Benzvix ${ }^{\circledR}$ and Hexvix ${ }^{\circledR}$, which exhibit improved skin penetration $(31,32)$.

Another PS used in PDT is temoporfin (Foscan ${ }^{\circledR}$ in Fig. 3). It belongs to the chlorin-based family of PDT PSs (32) and consists of a very simple chlorin structure able to be photoactivated at $650 \mathrm{~nm}$, where such a compound shows a significant molar absorption coefficient $\left(\varepsilon=3.9 \times 10^{4} \mathrm{M}^{-1} \mathrm{~cm}^{-1}\right)$. Moreover, it exhibits also a significant solubility and a suitable half-life in human plasma (45-65 h), both facilitating intravenous administration and subsequent redistribution. Temoporfin has been used in EU for treating certain head and neck cancer tumors, but patients can remain photosensitive several weeks after treatment, which is a common adverse effect in PDT treatments $(21,32)$. Other chlorin-based PDT PSs are verteporfin (Visudyne ${ }^{\circledR}$ ) and talaporfin sodium (Laserphyrin ${ }^{\circledR}$ ) (Fig. 3). Visudyne ${ }^{\circledR}$ (Fig. 3) is a self-aggregate able to be photoactivated at $689 \mathrm{~nm}$, clearing rapidly from the body and, consequently, giving place to shortterm skin photosensitivity. Visudyne ${ }^{\circledR}$ has been mainly used in age-related macular degeneration (8). Regarding Laserphyrin ${ }^{\circledR}$ (Fig. 3), it is an excellent ${ }^{1} \mathrm{O}_{2}$ generator, but its application is limited due to its high photobleaching rate.
On the other hand, several bacteriochlorins are highlighted as clinical important agents for PDT, such as water-soluble Pd-bacteriopheophorbide $\left(\right.$ Tookad $^{\circledR}$, WST 09 in Fig. 3), which exhibits strong light absorption $\left(\varepsilon=11 \times 10^{4} \mathrm{M}^{-1} \mathrm{~cm}^{-1}\right)$ into the spectral therapeutic window (absorption maximum at $763 \mathrm{~nm}$ ), as well as greater skin penetration in comparison with Photofrin ${ }^{\circledR}$ (4 $\mathrm{mm}$ versus $1.6 \mathrm{~mm}$ ) (22,31,32). Finally, the so-called third generation of PDT PSs is confirmed by certain second-generation PDT PSs conjugated to different carrier structures (e.g. monosaccharides, antibodies, nanoparticles and peptides) able to selectively transport the PSs into the tumor tissue $(21,23,31,32)$.

Trying to advance in the development of PDT PSs, other chromophoric scaffolds have been reported as promising candidates to overcome some of the drawbacks of the currently established PSs, mainly related to photobleaching, dark toxicity, low efficiency into the spectral therapeutic window, etc. In this line, phthalocyanines (Pcs) are structurally related to porphyrins, but Pcs show strong absorption in the red spectral region (Q-band), reaching the desired therapeutic window. This makes the Pc scaffold ideal for developing PDT PSs. Among Pcs, those containing diamagnetic metal ions, such as $\mathrm{Al}(\mathrm{III})$ or $\mathrm{Zn}$ (II) (Fig. 2), have been demonstrated to exhibit high ISC quantum yield and long triplet-state lifetime (9), both facilitating the efficient generation of ${ }^{1} \mathrm{O}_{2}$. Among metal complexes, those based on polypyridyl $\mathrm{Ru}$ (II) or Ir(III) (Fig. 2) highlight as the most studied dyes into this context $(7-8,33)$. Nonetheless, there is also evidence that other metal complexes, such as certain Pt(II) ones, could also serve as valuable scaffolds for the development of advanced ${ }^{1} \mathrm{O}_{2}$ PSs for PDT (8).

Another approach for the development of advanced PDT is using carbon-based nanostructures such as fullerenes (Fig. 2), $\mathrm{C}_{[60]}$ fullerene being the most stable, symmetrical and abundant fullerene. In addition, it shows high capability to photogenerate singlet oxygen $\left(\Phi_{\Delta}\right.$ near 1$)$ because of a high probability of intersystem crossing resulting in long-lasting triplets (hundreds of microseconds). However, $\mathrm{C}_{[60]}$ fullerene cannot be considered a good PDT photosensitizer by itself since it has low solubility in most solvents and absorbs mainly in the ultraviolet (UV) region $\left(\lambda_{\mathrm{abs}}<350 \mathrm{~nm}\right)$. However, it can be chemically modified to gain proper water solubility and ability to be excited in the Vis spectral region, the latter by designing multichromophoric systems in which the fullerene core acts as the acceptor chromophore (34). It must be noted here that $\mathrm{C}_{[60]}$ fullerene is a good "spin converter" of many chromophores, and by the said multichromophoric approach, the high singlet oxygen generation of $\mathrm{C}_{[60]}$ fullerene can be kept and triggered under Vis irradiation.

Finally, BODIPYs were revealed as valuable dyes for the development of different photonic applications due to their outstanding chemical and physical features (33-43). They show high absorption coefficients in the Vis region, high resistance to photobleaching (high photostability), low dependence on the photophysical signatures with the environmental conditions (e.g. solvent polarity), as well as small size and high lipophilicity, thus facilitating biological-membrane penetration. On the other hand, the high chemical versatility of the BODIPY core offers rich BODIPY chemistry, which allows an exhaustive and selective modification of the chromophoric BODIPY core (BODIPY functionalization), making possible the fine modulation of different properties, including key photophysical features (44). 


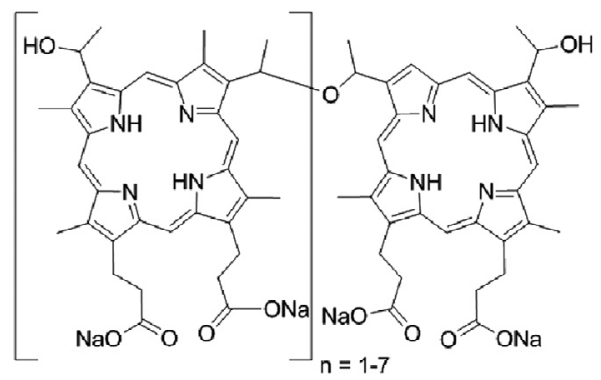

Oligomeric porphyrin units linked by ethers

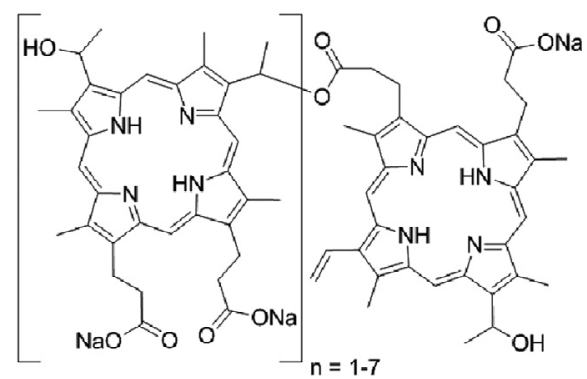

Oligomeric porphyrin units linked by esthers

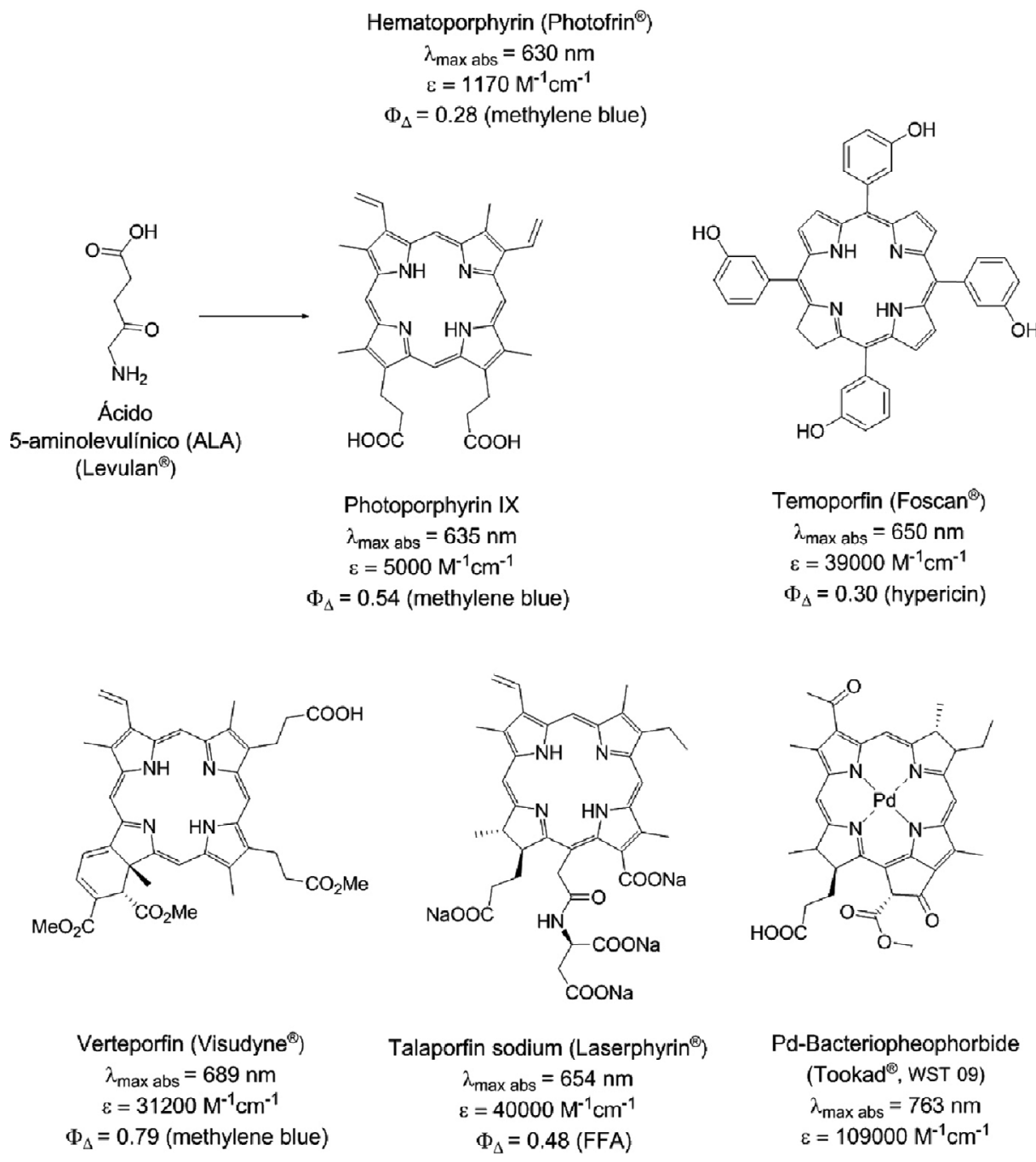

Figure 3. Some PDT PSs based on porphyrin.

Despite BODIPY dyes usually exhibit almost negligible efficiency for triplet $\left(T_{1}\right)$ population, as shown by their high fluorescence quantum yields, in this work we show some examples of how the fluorescent BODIPYs can be easily transformed in efficient PSs for PDT. In this context, several chemical approaches are applied to BODIPY dyes to make them able to generate ROS species, mainly ${ }^{1} \mathrm{O}_{2}$, by light irradiation $(8-12,16,45)$. For instance, ISC process populating $\mathrm{T}_{1}$ state for ${ }^{1} \mathrm{O}_{2}$ generation is easily promoted in BODIPYs by straightforwardly attaching heavy atoms (mainly iodine and bromine atoms, but also transition metals) to the BODIPY core (heavy-atom effect), thanks to the workable BODIPY chemistry. Another approach is based on the direct link of two BODIPY units in an orthogonal geometry (orthogonal BODIPY dimers). This last way is interesting for the development of PDT PSs based on BODIPY, as it avoids the inherent dark toxicity associated with heavy atoms $(9-11,46,47)$. Moreover, a suitable chemical modulation of the BODIPY structural scaffold opens avenues to finely balance ${ }^{1} \mathrm{O}_{2}$ 
photogeneration and fluorescence, which is an interesting property to develop PDT + bioimaging theragnostic agents (13), as well as to modulate other physical and chemical properties for an optimal photobiological activity (e.g. water solubility and membrane permeability) $(3,10,11)$.

Finally, it is worth to mention that combining well-known drug-carrier nanoparticles (gold, silica and polymeric) and PSs is a typical approach to improve solubility, and selectivity targeting and delivering of PSs for PDT applications, so offering a better control of the PS transportation and selective accumulation in the tumor tissues $(3,11,48)$. Moreover, the application of nanoparticles in cancer therapy and diagnosis has shown other advantages, including overcoming multidrug resistance and prevention of enzymatic degradation of the active agent (49). For all these reasons, the development of BODIPYs with reactive groups allowing their anchoring to nanoparticles is of special interest, and it is also described in this work (50-54).

\section{RESULTS}

\section{Advancing in the development of photosensitizers for ${ }^{1} \mathrm{O}_{2}$ based on BODIPY}

This minireview focuses on the contributions of our research group on the development of BODIPYs as ${ }^{1} \mathrm{O}_{2}$ PSs, which have been mainly achieved by two synthetic approaches: (1) attaching heavy atoms (mainly iodine and bromine, but also transition metals) to BODIPY scaffolds and (2) designing orthogonal BODIPY dimers (Fig. 4).

Halogenated BODIPYs. As mentioned above, the presence of heavy atoms increases the ISC quantum yield. Thus, it has been shown that BODIPY dyes with iodine or bromine atoms covalently linked to the BODIPY core can be promising PDT PSs, as an alternative to the porphyrin-based photosensitizers $(8-12,45)$; Nagano et al. (47) firstly reported an iodinated BODIPY for ${ }^{1} \mathrm{O}_{2}$ generation directed to PDT. In the same line, Akkaya (11-12,5561), Burgess $(10,46,62,63)$, Zhao $(8,64)$ and O'Shea $(65)$, among others (66-73), have also studied BODIPYs with different halogen substitution patterns for the same purpose.

Our contribution to the development of PSs based on haloBODIPYs has been to conduct a study on the influence of different factors (BODIPY basic structure, halogenated substitution pattern and nature of the involved halogens) on the ${ }^{1} \mathrm{O}_{2}$ generation efficiency, so establishing key factors controlling the design of future PDT PSs based on haloBODIPYs (74-77). The studied haloBODIPYs were based on 8-tolylBODIPY (Series A;

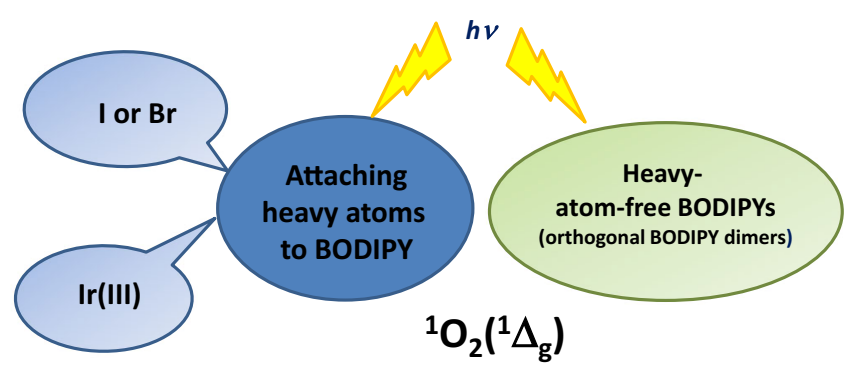

Figure 4. Diagram illustrating how the BODIPY scaffold can be adapted for ${ }^{1} \mathrm{O}_{2}$ photosensitization. compounds 2-8 in Fig. 5 and Table 1) (74,75), trialkylBODIPY (Series B; compounds $\mathbf{9 - 1 3}$ in Fig. 5 and Table 1) (75) and 8heteroatom-substituted BODIPY (Series C; compounds 14-17 in Fig. 5 and Table 1) (76). Additionally, BODIPYs 18 and 19 endowed with a reactive group (hydroxycarbonyl and trialkoxysilyl) and related to series $\mathrm{A}$ and $\mathrm{C}$, respectively, were designed for ulterior anchoring to nanoparticles (77).

From the conducted study, we observed that the number and position of halogen atoms strongly influence the location of the absorption and emission bands. Indeed, the successive halogenation of the BODIPY core gave rise to a progressive bathochromic shift of the spectral bands, except for the positions 1 and/or 7 (Table 1) (74). In fact, this effect is more pronounced by iodination (compounds 6-8, Table 1) than by bromination (compounds 2-5, Table 1). For instance, tetraiodination in compound 8 makes this dye to show spectral bands near the red region of the Vis spectrum (ca. 580/595 $\mathrm{nm}$ for absorption/emission).

On the other hand, an increased number of heavy atoms do not necessarily increase ${ }^{1} \mathrm{O}_{2}$ production. Thus, in Series A, we observed that the highest ${ }^{1} \mathrm{O}_{2}$ production is reached when the halogen atoms are attached to the 2 and 6 positions of the BODIPY structure (see brominated $\mathbf{2 - 5}$ and iodinated 6-8 in Fig. 5 and Table 1), whereas extra halogenation at positions 3 and/or 5 does not produce a significant increase in ${ }^{1} \mathrm{O}_{2}$ production but, noticeably, favors fluorescence efficiency (see $\mathbf{4}, \mathbf{5}$ and $\mathbf{8}$ in Fig. 5 and Table 1).

In the case of asymmetric alkyl substitution (Series B), a beneficial effect regarding ${ }^{1} \mathrm{O}_{2}$ production was observed when the halogen atoms are located at the 1 position of BODIPY. Indeed, compounds 9 and 11, having two identical heavy halogens at positions 1 and 2, show higher $\Phi_{\Delta}$ than analogous $\mathbf{1 0}$ and $\mathbf{1 2}$ having those atoms at positions 2 and 3 (Fig. 5 and Table 1). In this line, a detrimental effect on ${ }^{1} \mathrm{O}_{2}$ production is observed by halogen substitution at position 3 (see $\mathbf{1 3}$ in Fig. 5 and Table 1), regardless of the presence of the beneficial halogen substitution at 1 . These results demonstrate the importance of controlling the position of the halogen substitution when designing potential PDT PSs based on BODIPY.

On the other hand, the type of heavy halogen atom on the BODIPY core exerts an important impact on both ${ }^{1} \mathrm{O}_{2}$ generation and fluorescence. Thus, it was evidenced that iodinated 6-8, 15 and 17 (see Table 1) show higher $\Phi_{\Delta}$ values than their brominated counterparts (2-5 and $\mathbf{1 6}$ in Table 1). This fact is due to the higher spin-orbit coupling induced by iodine since it depends on the fourth power of the atom orbital quantum number, allowing a more efficient ISC than the substitution by bromine atoms. Thus, BODIPYs with two iodines at positions 2 and 6 are characterized by very high ${ }^{1} \mathrm{O}_{2}$ production $\left(\Phi_{\Delta} \geq 0.85\right.$ ) (see $\mathbf{6}, 15$ and 17, Table 1) and low fluorescence quantum yield $\left(\Phi_{\text {flu }} \leq 0.05\right)$. However, related 2,6-dibrominated BODIPYs show $\Phi_{\Delta} \sim 0.60$ (see 2 and 16, Table 1), together with a moderate emission quantum yield $\left(\Phi_{\text {flu }} \sim 0.30\right)$ offering an interesting dual photonic action with a balanced fluorescence emission and ${ }^{1} \mathrm{O}_{2}$ generation. Consequently, these brominated BODIPYs could be used as convenient platforms for developing theragnostic PDT + bioimaging agents. However, if a strong therapeutic activity is required (by maintaining low dye doses), iodination at positions $2 / 6$ should be the best choice for the haloBODIPY design.

Regarding heteroatom substitution in the position 8 of the BODIPY structure, it markedly affects the location of the 
<smiles></smiles>
1: $R^{1}=H \quad R^{7}=H \quad R^{2}=H \quad R^{6}=H \quad R^{3}=H \quad R^{5}=H$
2: $\mathrm{R}^{1}=\mathrm{H} \quad \mathrm{R}^{7}=\mathrm{H} \quad \mathrm{R}^{2}=\mathrm{Br} \quad \mathrm{R}^{6}=\mathrm{Br} \quad \mathrm{R}^{3}=\mathrm{H} \quad \mathrm{R}^{5}=\mathrm{H}$
3: $\mathrm{R}^{1}=\mathrm{H} \quad \mathrm{R}^{7}=\mathrm{H} \quad \mathrm{R}^{2}=\mathrm{H} \quad \mathrm{R}^{6}=\mathrm{H} \quad \mathrm{R}^{3}=\mathrm{Br} \quad \mathrm{R}^{5}=\mathrm{Br}$
4: $\mathrm{R}^{1}=\mathrm{H} \quad \mathrm{R}^{7}=\mathrm{H} \quad \mathrm{R}^{2}=\mathrm{Br} \quad \mathrm{R}^{6}=\mathrm{Br} \quad \mathrm{R}^{3}=\mathrm{Br} \quad \mathrm{R}^{5}=\mathrm{Br}$
5: $\mathrm{R}^{1}=\mathrm{Br} \quad \mathrm{R}^{7}=\mathrm{Br} \quad \mathrm{R}^{2}=\mathrm{Br} \quad \mathrm{R}^{6}=\mathrm{Br} \quad \mathrm{R}^{3}=\mathrm{Br} \quad \mathrm{R}^{5}=\mathrm{Br}$
6: $R^{1}=H \quad R^{7}=H \quad R^{2}=I \quad R^{6}=1 \quad R^{3}=H \quad R^{5}=H$
7: $\mathrm{R}^{1}=\mathrm{H} \quad \mathrm{R}^{7}=\mathrm{H} \quad \mathrm{R}^{2}=\mathrm{I} \quad \mathrm{R}^{6}=\mathrm{I} \quad \mathrm{R}^{3}=\mathrm{I} \quad \mathrm{R}^{5}=\mathrm{H}$

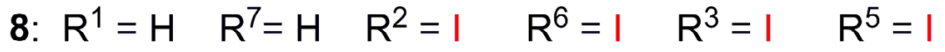<smiles></smiles>
9: $\mathrm{R}^{1}=\mathrm{Br} \mathrm{R}^{2}=\mathrm{Br} \quad \mathrm{R}^{3}=\mathrm{H}$
10: $R^{1}=H \quad R^{2}=B r \quad R^{3}=B r$
11: $R^{1}=I \quad R^{2}=I \quad R^{3}=H$
12: $R^{1}=H \quad R^{2}=I \quad R^{3}=1$
13: $R^{1}=I \quad R^{2}=I \quad R^{3}=I$<smiles></smiles>
14: $\mathrm{R}^{8}=\mathrm{NHMe}$
$\mathrm{R}^{2}=\mathrm{I} \quad \mathrm{R}^{6}=\mathrm{I}$
15: $R^{8}=$ NH-propynyl
$\mathrm{R}^{2}=\mathrm{I} \quad \mathrm{R}^{6}=\mathrm{I}$
16: $\mathrm{R}^{8}=\mathrm{SMe}$
$\mathrm{R}^{2}=\mathrm{Br} \mathrm{R}^{6}=\mathrm{Br}$
17: $R^{8}=S M e$
$\mathrm{R}^{2}=\mathrm{I} \quad \mathrm{R}^{6}=$ I
18: $\mathrm{R}^{8}=\mathrm{C}_{6} \mathrm{H}_{4}-\mathrm{COOH}$
$\mathrm{R}^{2}=\mathrm{I} \quad \mathrm{R}^{6}=\mathrm{I}$
19: $\mathrm{R}^{8}=\mathrm{NH}-\left(\mathrm{CH}_{2}\right)_{3}-\mathrm{Si}(\mathrm{OEt})_{3} \quad \mathrm{R}^{2}=1 \quad \mathrm{R}^{6}=1$

Figure 5. Halogenated BODIPYs studied by our research group as PSs (see Table 1).

maximum of the spectral (absorption/emission) bands, which depends on the nature of the heteroatom attached to the meso position of the haloBODIPY and, particularly, on the electronegativity of the involved heteroatom, as previously described for nonhalogenated BODIPYs (78). Thus, 8-aminoBODIPYs 14 and 15 gave a very pronounced blueshift (ca. $100 \mathrm{~nm}$ ) when compared with related 8-(methylthio)BODIPY 17, in agreement with the Hammett $\sigma$-parameter of the groups located at position 8 $\left(\sigma_{\mathrm{p}}^{+}=-1.81\right.$ for NHMe and ${\sigma_{\mathrm{p}}}^{+}=-0.60$ for SMe) (76). This effect is mainly attributed to the high contribution of a hemicyanine-like BODIPY structure in the case of the aminated derivatives (78-80). Indeed, the higher the electron-donor capacity of the amine is, the higher the blueshift is, as it is shown by BODIPY 14 with a methyl group $\left(\sigma_{\mathrm{p}}{ }^{+}=-0.31\right)$ involved in the 8(methylamino) moiety, in comparison with a propargyl one (with \left. lower electron-donor strength; ${\sigma_{\mathrm{p}}}^{+}=+0.18\right)$ involved in the 8(propargylamino) group of BODIPY 15 (Table 1). Note here that, the different substitution in the amino group in these halogenated 8-aminoBODIPYs also affects the production of ${ }^{1} \mathrm{O}_{2}$. Although it is well known, the ability of aliphatic amines, mainly tertiary, to efficiently quench ${ }^{1} \mathrm{O}_{2}$ via partial charge-transfer intermediates (15), the singlet oxygen production quantum yield of BODIPY 15 very close to one, practically discards this effect. However, the higher electron-donor capacity of the amine group of BODIPY 14 decreases ${ }^{1} \mathrm{O}_{2}$ production $\left(\Phi_{\Delta}=0.78\right.$ vs
$\Phi_{\Delta}=0.96$, for 14 and 15, respectively, Table 1). This fact can be attributed to a more favored physical quenching of ${ }^{1} \mathrm{O}_{2}$ but also to the activation of an intramolecular charge transfer (ICT) between the amine and the BODIPY, which competes with the radiative emission from the singlet state (fluorescence) and the intersystem crossing pathway to the triplet state (eventually leading to singlet oxygen production). Nonetheless, both halogenated 8-aminoBODIPYs (14 and 15) are potentially interesting for the development of PSs, upon blue or UV irradiation, respectively, due to their high ability to produce ${ }^{1} \mathrm{O}_{2}$.

Accordingly, halogenated 8-(methylthio)BODIPYs 16 and 17 exhibit hypsochromic shift of the absorption band in comparison with their parent structures $\mathbf{2}$ and $\mathbf{6}$, respectively, this blueshift being lower than that occurring in related 8-aminoBODIPYs 14 and 15 (see Fig. 5 and Table 1). However, the most relevant aspect of these 8-(methylthio)BODIPYs, particularly in the case of $\mathbf{1 7}$, is that they were revealed as good standards for the quantification of ${ }^{1} \mathrm{O}_{2}$ production by irradiation in the $\mathrm{UV}$ and $\mathrm{Vis}$ region. In this context, although $\mathrm{PN}$ is considered the universal reference for quantifying $\Phi_{\Delta}$, with $\Phi_{\Delta}$ close to 1 in both polar and nonpolar solvents, but only for the UV spectral range (20). On the other hand, as it was mentioned above, the references used in the Vis region, such as $\mathrm{RB}$ or $\mathrm{MB}$, are only suitable in polar solvents such as $\mathrm{ACN}$, EtOH or $\mathrm{MeOH}$, and very scattered $\Phi_{\Delta}$ values are reported in the literature (i.e. $\Phi_{\Delta}=0.5-0.8$ for RB 
Table 1. Photophysical properties of halogenated BODIPYs in air-equilibrated ACN.

\begin{tabular}{ccclll}
\hline Compound & $\lambda_{\text {abs }}(\mathrm{nm})$ & $\varepsilon_{\max }\left[\mathrm{M}^{-1} \mathrm{~cm}^{-1}\right]$ & $\lambda_{\text {flu }}(\mathrm{nm})$ & $\Phi_{\text {flu }}$ & $\Phi_{\Delta}$ \\
\hline $\mathbf{1}$ & 550 & 69000 & 516 & 0.04 & 0 \\
$\mathbf{2}$ & 531 & 46200 & 554 & 0.33 & $0.57^{*}$ \\
$\mathbf{3}$ & 515 & 75700 & ND & ND & $0.26^{*}$ \\
$\mathbf{4}$ & 546 & 57300 & ND & ND & $0.58^{*}$ \\
$\mathbf{5}$ & 544 & 70500 & ND & ND & $0.47^{*}$ \\
$\mathbf{6}$ & 548 & 43000 & 567 & 0.01 & $0.83^{*}$ \\
$\mathbf{7}$ & 573 & 48000 & 577 & 0.06 & $0.86^{*}$ \\
$\mathbf{8}$ & 581 & 43800 & 593 & 0.10 & $0.94^{*}$ \\
$\mathbf{9}$ & 488 & 31300 & ND & ND & $0.90^{*}$ \\
$\mathbf{1 0}$ & 509 & 33100 & ND & ND & $0.60^{*}$ \\
$\mathbf{1 1}$ & 503 & 24400 & ND & ND & $0.99^{*}$ \\
$\mathbf{1 2}$ & 521 & 28800 & ND & ND & $0.60^{*}$ \\
$\mathbf{1 3}$ & 525 & 33900 & ND & ND & $0.60^{*}$ \\
$\mathbf{1 4}$ & 426 & 39000 & 484 & 0.02 & $0.78 \dagger$ \\
$\mathbf{1 5}$ & 440 & 38000 & 521 & 0.05 & $0.96 \dagger$ \\
$\mathbf{1 6}$ & 527 & 42000 & 576 & 0.33 & $0.61^{*}$ \\
$\mathbf{1 7}$ & 533 & 43000 & 588 & 0.04 & $0.85^{*}$ \\
$\mathbf{1 8}$ & 550 & 73000 & 578 & 0.03 & $0.66^{*}$ \\
$\mathbf{1 9}$ & - & - & - & - & -
\end{tabular}

*Reference is $\mathrm{RB}$ in $\mathrm{ACN}\left(\Phi_{\Delta}=0.53\right)$; treference is $\mathrm{PN}$ in $\mathrm{ACN}$ $\left(\Phi_{\Delta}=1\right)(19)$; $\$$ Photophysical properties were assumed to be similar to those of its homologous 14.

in $\mathrm{ACN}$ ) (1). In this regard, compound 17 shows a high $\Phi_{\Delta}$ under UV or Vis excitation $(300-400$ or $500-600 \mathrm{~nm}$, $\Phi_{\Delta}>0.87$ ), irrespective of the polarity of the solvent together with a high photostability and negligible deactivation of singlet oxygen by the sensitizer itself.

Finally, the inclusion of reactive functionalities in haloBODIPYs (e.g. see 18 and 19 in Fig. 5), without significantly interfering with the production of ${ }^{1} \mathrm{O}_{2}$, could be interesting for their immobilization onto different nanocarriers (76,81-83). As an example of biocompatible nanomaterials, porous silica nanoparticles have demonstrated suitable features for advanced applications in biomedicine, such as large specific surface areas, controllable particle sizes and pore volumes, and straightforward functionalization of the external surface (84-86). In this context, core-shell amino-functionalized silica nanoparticles, having free silanol groups as well, were synthesized by us by sol-gel process (Fig. 6A; size ca. $50 \mathrm{~nm}$ ) and decorated with BODIPYs 18 and 19 (Fig. 6B-C), respectively, to be used as potential PS nanocarriers for PDT applications. Noticeably, the immobilized BODIPY-based PSs prepared by this method rendered higher $\Phi_{\Delta}$ values than those obtained by similar immobilization of which was attributed to the different aggregation capability of the functionalized nanoparticles, which strongly depends on the structure of the grafted PS and the grafting method.

Therefore, our studies on haloBODIPYs offer interesting platforms for the development of advanced PSs for PDT due to their easy tuning of key physical and chemical properties, making also possible their straightforward immobilization on different solid supports, such a silica nanoparticles, to generate functional nanomaterials directed to improve properties such as biodistribution or bioselectivity, of interest in biomedical applications.

BODIPY-involving Ir(III) complexes. Another interesting example of the use of BODIPY dyes as efficient PSs for the generation of ${ }^{1} \mathrm{O}_{2}$ aiming to their application in photomedicine is the chelation of a BODIPY-based ligand to a metal center. Indeed, many organometallic complexes containing transition metal ions, such as $\mathrm{Ru}(\mathrm{II})$, Os(II) or $\mathrm{Ir}(\mathrm{III})$, turned out as efficient PSs for ${ }^{1} \mathrm{O}_{2}$ production. These compounds offer the possibility of independently regulating both their emissive properties and their ${ }^{1} \mathrm{O}_{2}$ production by modifying the involved organic ligands (87). However, although some PSs based on Ir(III) complexes with 2,2'-bipyridine (bpy) have demonstrated efficient ${ }^{1} \mathrm{O}_{2}$ photogeneration, they mostly showed absorption bands in the UV region with low molar absorption coefficients, limiting their use in PDT applications $(8,88)$. In order to overcome this drawback, several designing strategies have been followed by attaching light-harvesting chromophores to the coordination complex (88-90), which can be also promising to achieve dyes with combined fluorescence and photosensitization features for simultaneous imaging and PDT.

In this line, different cyclometalated complexes having BODIPY ligands chelated to the metal center have been already described (88-92). The type of linker connecting the light-harvesting chromophore (i.e. the BODIPY moiety) as well as the metal center is key selection to achieve efficiency in the energy funneling toward the excited triplet-state manifold. Lots of works have focused on connecting the metallic center to the Vis-absorbing chromophore by $\pi$-conjugated linkers, generally through 2,2'-bipyridine chelating connectors (Fig. 7). On the other hand, dye-functionalized PS complexes have been also synthesized by directly attaching the Vis-absorbing chromophore to the metal center, mainly through acetylide units (see Fig. 7B). However, the efficiency of the majority of these compounds as PDTbioimaging agents remains low.

Taking into account the previous work on $\operatorname{Ir}(\mathrm{III})$ complexes containing a BODIPY moiety, we described a new designing strategy to develop advanced PDT-bioimaging agents based on biscyclometalated Ir(III) complexes involving a BODIPY-based ancillary ligand, where the BODIPY unit is directly grafted to the metal center by acetylacetonate (acac) chelating group located at the BODIPY meso position (20 and 21, Fig. 8). To elucidate the relevance of the involved acac-BODIPY moiety in the efficiency as a PS of these novel dyes, a related iridium complex bearing the same biscyclometalated Ir(III) unit and mesosubstituted BODIPY moiety, but connected through a large, dipyridophenazine $\pi$-conjugated ligand (22 in Fig. 8), was also synthesized and studied (93).

The attachment of acac-BODIPY to Ir(III) does not result in any noticeable electron coupling between the involved chromophores, at least in the ground state, since the absorption spectra of $\mathbf{2 0}$ and $\mathbf{2 1}$ are roughly the combination of the absorption spectra of each isolated moieties (Fig. 8). In this regard, no significant shift of the absorption bands upon complexation with $\mathrm{Ir}$ (III) was observed when compared to the electronically isolated BODIPY moieties (23 and $\mathbf{2 4}$ in Fig. 8). However, the absorption coefficients $(\varepsilon)$ at the wavelength of the absorption maximum underwent an eightfold increase, likely due to the replacement of the BODIPY fluorines by alkoxyls in these complexes, replacement which was also responsible for the improvement of their solubility in aqueous media.

The presence of thienyl groups at the positions 2 and 6 of BODIPY 21 induces an important shift of the absorption and fluorescence bands when compared to those of 20, placing its spectroscopic bands within the therapeutic window. Moreover, 21 shows an interesting balance between ${ }^{1} \mathrm{O}_{2}$ generation and 

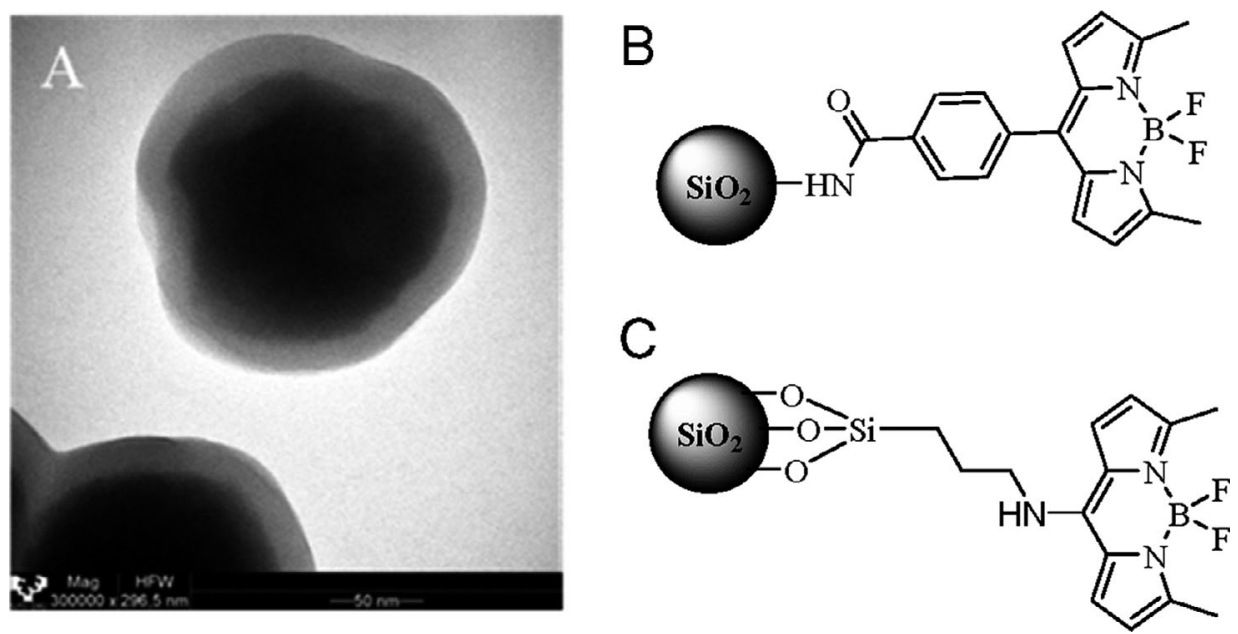

Figure 6. TEM image of a core-shell amino-functionalized silica nanoparticle (A). Schematic representation of the different BODIPY linkers used in such nanoparticles: by peptide-like coupling with 18 (B) or by direct polymerization with 19 (C).

A

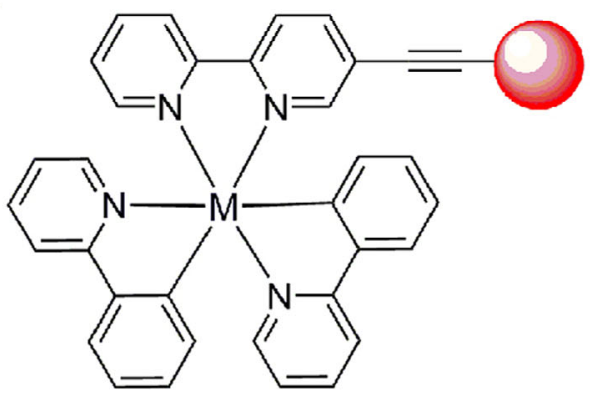

B<smiles></smiles><smiles></smiles>

Figure 7. Different examples of tethering a dye (red ball) to metal complexes. The metal center (M) is generally $\operatorname{Ir}(\mathrm{III})$ or $\mathrm{Ru}(\mathrm{II})$ in structure (A), or Pt (II) in structure B.

fluorescence under red light excitation, which makes it a promising scaffold for the development of theragnostic bioimaging + PDT applications.

On the other hand, complex 22 shows strong absorption in the Vis region, but its fluorescence is practically quenched and, more importantly, lacks the ability to generate ${ }^{1} \mathrm{O}_{2}$ by excitation with visible light. Indeed, 22 shows a moderate $\Phi_{\Delta}(51 \%)$, but only by excitation of the metal chromophore under UV irradiation $(370 \mathrm{~nm})$. The different behavior of $\mathbf{2 0}$ and $\mathbf{2 1}$, with respect to $\mathbf{2 2}$ demonstrates the importance of the linker when designing PDT PSs based on these dye-involving metal complexes.

The interesting PS capability and fluorescence of $\mathbf{2 0}$ and $\mathbf{2 1}$ prompted us to conduct an in vitro study on the PDT and bioimaging activity of these compounds by using HeLa cells, in order to analyze their capacity as potential theragnostic agents. By incubating the cells with the corresponding dye for $24 \mathrm{~h}$, both compounds exhibited good cell internalization, as visualized by fluorescence microscopy (Fig. 9A).

Interestingly, both compounds triggered phototoxicity on HeLa cells without exhibiting dark toxicity (i.e. they are not cytotoxic in the absence of light irradiation, as shown in Fig. 9B). However, apoptotic cells were seen after photodynamic treatment with green excitation centered at $532 \mathrm{~nm}$ for $\mathbf{2 0}$ or at $632 \mathrm{~nm}$ for 21 (Fig. 9D). Note here that $\mathbf{2 0}$ showed a significantly higher phototoxicity than 21 (Fig. 9C), triggering $50 \%$ of cell death at very low dye concentration $(50 \mathrm{nM}$ for $\mathbf{2 0})$ as a consequence of its higher capacity to generate singlet oxygen $\left(\Phi_{\Delta}=0.86 v s \Phi_{\Delta}=0.60\right.$, for $\mathbf{2 0}$ and $\mathbf{2 1}$, respectively). Therefore, the obtained results (cell signalization by fluorescence and PDT) demonstrate the potential of the BODIPY-based iridiumcomplex design for developing theragnostic agents based on PDT and fluorescence bioimaging. 


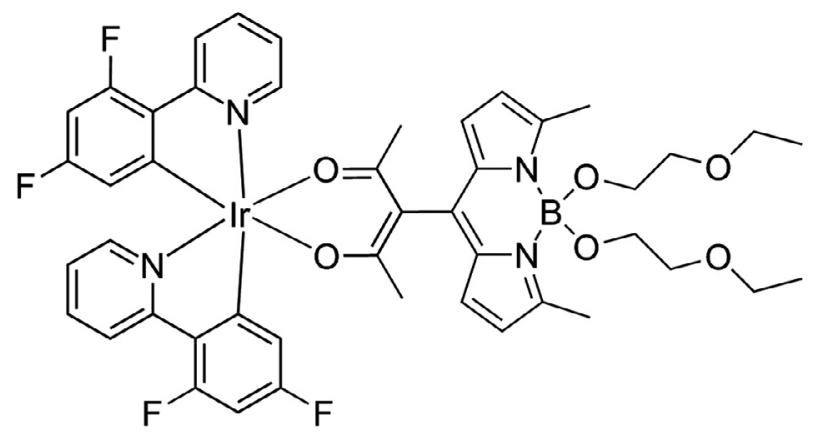

20

$\lambda_{\text {abs }}=517 \mathrm{~nm}$

$\lambda_{\text {flu }}=528 \mathrm{~nm}$

$\varepsilon_{\max }=4.46 \cdot 10^{4} \mathrm{M}^{-1} \mathrm{~cm}^{-1}$

$\Phi_{\text {flu }}=0.08$

$\Phi_{\Delta}=0.86\left(\lambda_{\text {exc }}=530 \mathrm{~nm}\right.$,

rose Bengal as reference)

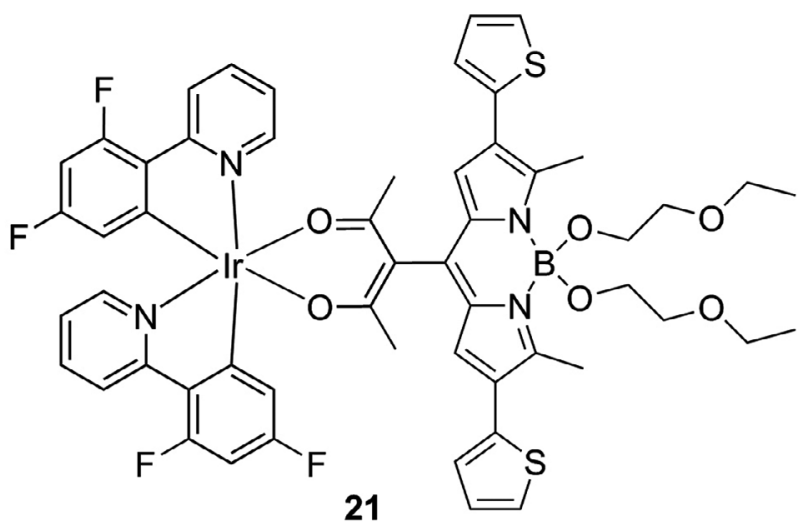

$\lambda_{\text {abs }}=597 \mathrm{~nm}$

$\lambda_{\text {flu }}=652 \mathrm{~nm}$

$\varepsilon_{\text {max }}=3.38 \cdot 10^{4} \mathrm{M}^{-1} \mathrm{~cm}^{-1}$

$\Phi_{\text {flu }}=0.22$

$\Phi_{\Delta}=0.60\left(\lambda_{\text {exc }}=605 \mathrm{~nm}\right.$, methylene blue as reference)

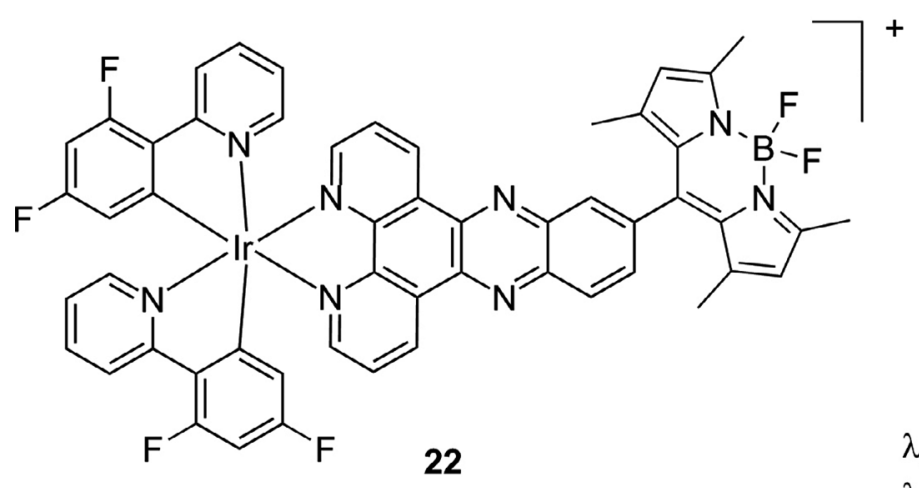<smiles></smiles>

23

$\lambda_{\text {abs }}=515 \mathrm{~nm}$ $\lambda_{\mathrm{fl}}=526 \mathrm{~nm}$

$\lambda_{\mathrm{abs}}=502 \mathrm{~nm}$

$\lambda_{\mathrm{flu}}=546 \mathrm{~nm}$

$\varepsilon_{\text {max }}=2.60 \cdot 10^{4} \mathrm{M}^{-1} \mathrm{~cm}^{-1}$

$\Phi_{\text {flu }}=0.01$

$\Phi_{\Delta}=0.51\left(\lambda_{\text {exc }}=370 \mathrm{~nm}\right.$,

phenalenone as reference) $\Phi_{\text {flu }}=0.90$<smiles></smiles>

24 $\lambda_{\text {flu }}=677 \mathrm{~nm}$ $\varepsilon_{\max }=0.99 \cdot 10^{4} \mathrm{M}^{-1} \mathrm{~cm}^{-1} \quad \varepsilon_{\max }=0.41 \cdot 10^{4} \mathrm{M}^{-1} \mathrm{~cm}^{-1}$ $\Phi_{\text {flu }}=0.09$

Figure 8. Structures of the iridium complexes (20-22) and free acac-BODIPY (23-24).

Orthogonal BODIPY dimers. Although heavy atoms can efficiently promote ISC, they might also increase the intrinsic dark toxicity of the PS, limiting its application for clinical PDT $(45,46)$. As an alternative, Flamigni et al. reported the first heavy-atom-free ${ }^{1} \mathrm{O}_{2}$ PSs based on orthogonal BODIPY dimers $(94,95)$. In fact, such an orthogonal disposition of both chromophores has been pointed out as an elegant way to attain ${ }^{1} \mathrm{O}_{2}$ generation avoiding the use of heavy halogen or metal atoms and their inherent toxicity. This appealing approach drew the attention of several research groups, due to its added value for developing biomedical applications (96101).

We have also developed and studied a series of orthogonal BODIPY dimers, including $\pi$-extended functionalization with the aim of shifting the absorption band to the red region of the Vis spectra (Fig. 10) (12). Such a study allowed us to rationally design a multichromophoric system with panchromatic action, based only on BODIPY chromophores, able to act as a low-toxic agent with dual (fluorescence bioimaging and PDT) functionality (13). 
A
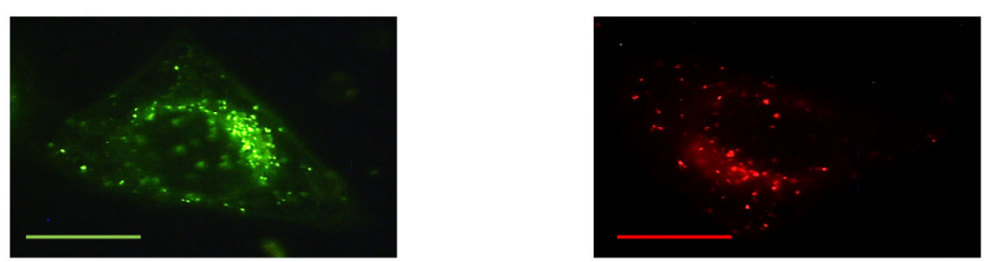

$\mathbf{B}$
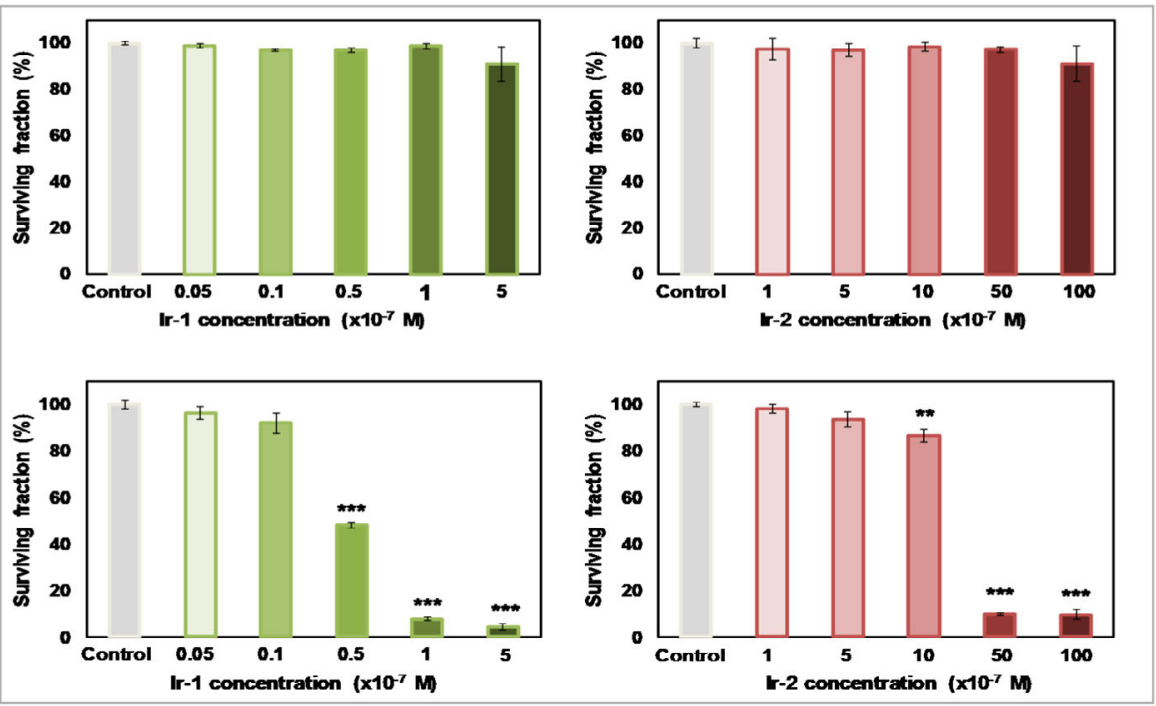

C

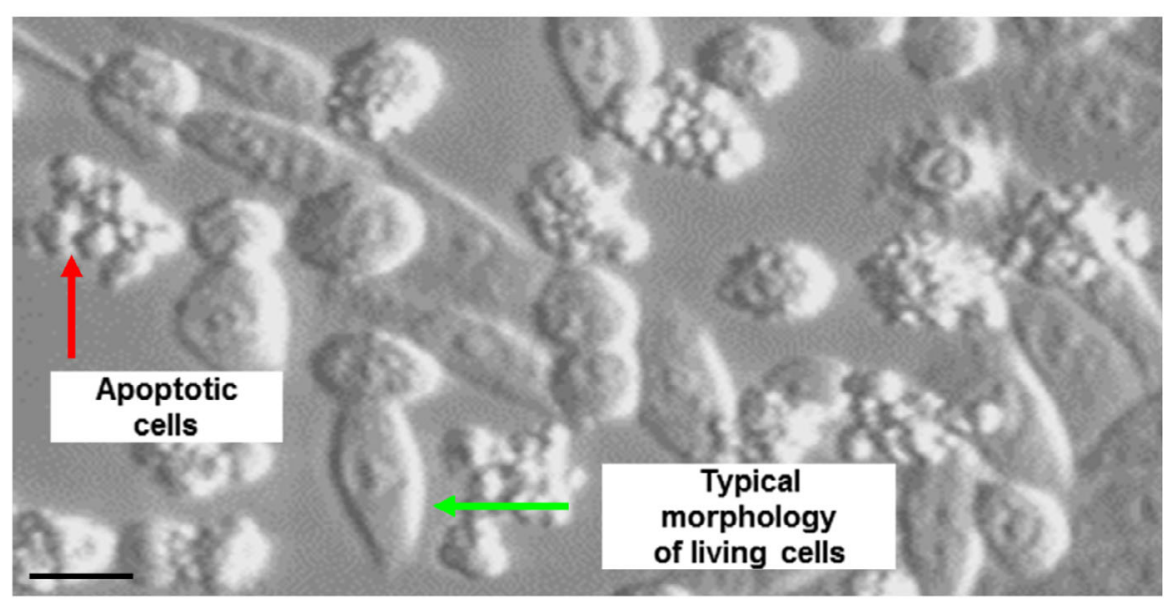

Figure 9. HeLa cells experiments. (A) Internalization of 20 and 21 in HeLa cells after incubation for $24 \mathrm{~h}$ with $5 \times 10^{-7} \mathrm{M}$ dye; scale bar: $10 \mu \mathrm{m}$. (B) Surviving fractions of cells after 24-h incubation with the corresponding dye at different concentrations in the absence of irradiation and (C) after exposition to $6.8 \mathrm{~J} \mathrm{~cm}^{-2}$ of green or red light. Cell viability was evaluated by MTT assay $24 \mathrm{~h}$ after each treatment. Data correspond to mean \pm SD values from at least six different experiments. Statistically significant differences are labeled as $*(P<0.01)$, ** $(P<0.005)$ and $* * *(P<0.001)$ for comparisons between groups using one-way ANOVA. (D) Image of HeLa cells obtained by inverted differential interference contrast (DIC) microscope after the photodynamic treatment with $\mathbf{2 1}$ (apoptotic cells display characteristic cellular changes, including cell shrinkage and plasma membrane blebbing (red arrow) compared to viable cells (green arrow). Scale bar: $10 \mu \mathrm{m}$ ).

The simplest dimer, compound $\mathbf{2 5}$, exhibited a very narrow (Fig. 11A) and intense absorption band with an absorption coefficient twofold higher $\left(\varepsilon>15 \times 10^{4} \mathrm{M}^{-1} \mathrm{~cm}^{-1}\right)$ than that of its parent monomeric BODIPY $\left(\varepsilon \sim 8 \times 10^{4} \mathrm{M}^{-1} \mathrm{~cm}^{-1}\right)$, indicating that both BODIPY units are electronically decoupled, because of the orthogonal disposition of the chromophores. Such orthogonal geometry was confirmed by computational calculations (Fig. 11B). Accordingly, DFT simulations predicted two nearly degenerated states with very similar oscillator strengths, where each BODIPY unit is excited independently (Fig. 11B). Remarkably, although the absorption band is nearly independent of the nature of the solvent, the fluorescence properties are significantly modified by solvent polarity (Fig. 11). As the solvent polarity increases, the fluorescence efficiency from the main emission band drastically decreases and, in polar solvents such as acetone and ACN, a new and broad redshifted band is observed 


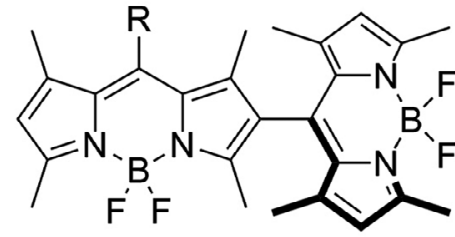

25: $\mathrm{R}=\mathrm{Me}$

26: $R=4$-nitrophenyl 27: $\mathrm{R}=4$-aminophenyl

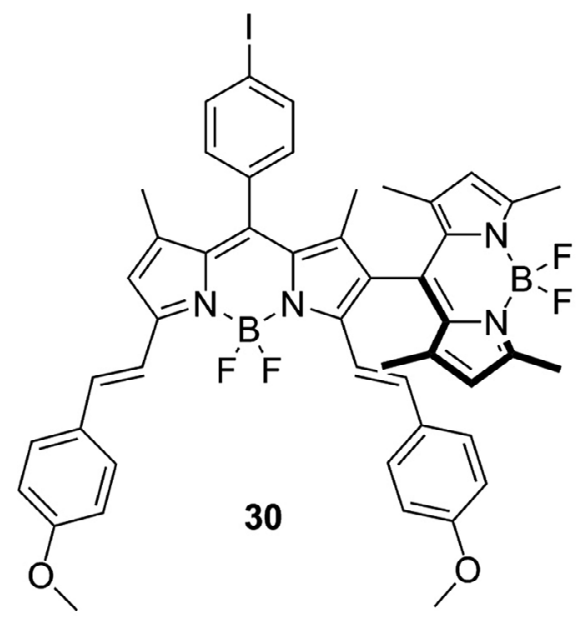<smiles></smiles>

28: $R$ = phenylethynyl 29: $R=2$-thienyl

Figure 10. Orthogonal BODIPY dimers as PSs (see Table 2) studied by Ortiz et al.

(Fig. 11A), which is characteristic of an ICT state. Regarding the computational studies performed for $\mathbf{2 5}$ in ACN (polar solvent), the same spatial distribution of their HOMO and HOMO-1 orbitals was shown, as it also occurs in less polar solvents such as chloroform $\left(\mathrm{CHCl}_{3}\right)$ or tetrahydrofuran (THF). However, in agreement with the study conducted by Thompson et al. (14), the LUMO and LUMO+1 are delocalized through both BODIPY units in polar solvents (Fig. 11B), allowing the population of an ICT state by solvent-induced symmetry breaking in the excited state.

Importantly, the polarity of the solvent has also a big impact on the ability for generating ${ }^{1} \mathrm{O}_{2}$. Actually, as the polarity of the solvent increases, compound $\mathbf{2 5}$ gradually decreases its fluorescence efficiency with detrimental effects on ${ }^{1} \mathrm{O}_{2}$ photoproduction, which reaches its maximum for solvents of intermediate polarity (Fig. 11C). However, further increase in the solvent polarity leads to a reduction of both fluorescence and the ${ }^{1} \mathrm{O}_{2}$ generation (Fig. 11C).

In this regard, different theories about the photosensitization mechanism in orthogonal BODIPY dimers have been proposed (93-105). Thus, the experimental and computational studies conducted by Akkaya et al. (96) assigned the high ISC quantum yield of the orthogonal BODIPY dimers to the formation of degenerated HOMOs and LUMOs, as a consequence of the orthogonal arrangement of similar subunits in which the $\pi$ mixing is avoided. Thus, ${ }^{1} \mathrm{O}_{2}$ generation should decrease if either the orthogonal disposition of both units is disturbed or unsymmetrical dimer is formed. However, this hypothesis does not explain the high dependence on ${ }^{1} \mathrm{O}_{2}$ production with the solvent polarity. An alternative explanation for the efficiency of the orthogonal BODIPY dimers as PSs for ${ }^{1} \mathrm{O}_{2}$ production is the triplet-state population taking place from ICT states generated by charge recombination (ICT transition) in the singlet excited state (16-108). Nevertheless, the dependence of ${ }^{1} \mathrm{O}_{2}$ generation of halogen-free orthogonal BODIPY dimers on solvent polarity is in good agreement with a spin-orbit chargetransfer intersystem crossing (SOCT-ISC) mechanism $(12,109)$, which basically operates in orthogonal donor-acceptor dyads. Another plausible explanation for the formation of triplet states of $\mathbf{2 5}$ has been recently proposed by us (110). The measurements performed at a short timescale revealed that triplet states are formed in a few picoseconds with efficiencies near $100 \%$. This observation suggests exciton singlet fission (SF) as a possible mechanism (111-114), a spin-allowed process whereby a singlet excited state converts to two triplet states. This SF process depends on the solvent polarity, which points to the involvement of a charge-transfer-character state as stated before.

In this line, other orthogonal BODIPY dimers with different donor/acceptor substitution patterns were analyzed to study their impact on ${ }^{1} \mathrm{O}_{2}$ generation (12). Thus, the presence of an electron-withdrawing 4-nitrophenyl group in one of the monomer units (26 in Fig. 10) led to further stabilization of the ICT state, and therefore, the ${ }^{1} \mathrm{O}_{2}$ generation decreased with respect to that exhibited by dimer $25\left(\Phi_{\Delta}=0.43\right.$ vs 0.75 in $\mathrm{CHCl}_{3}$, Table 2). Conversely, the presence of an electron-donating 4-aminophenyl 
A

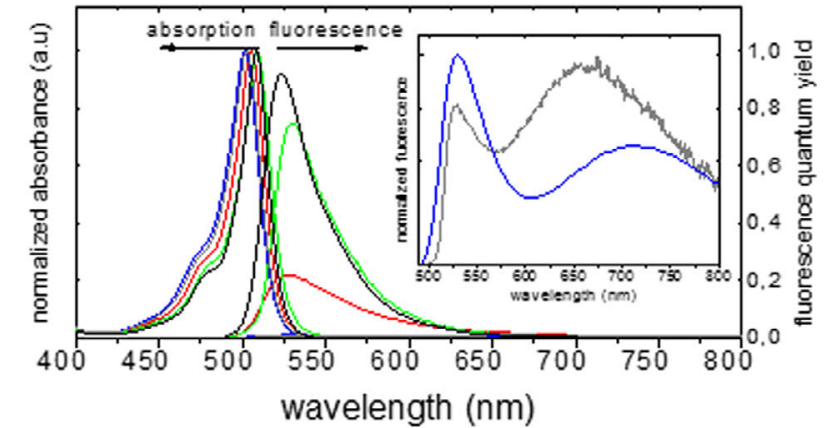

B

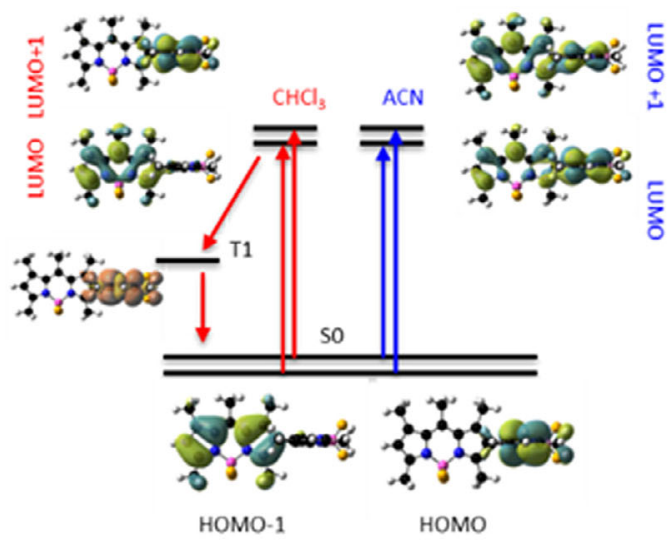

C

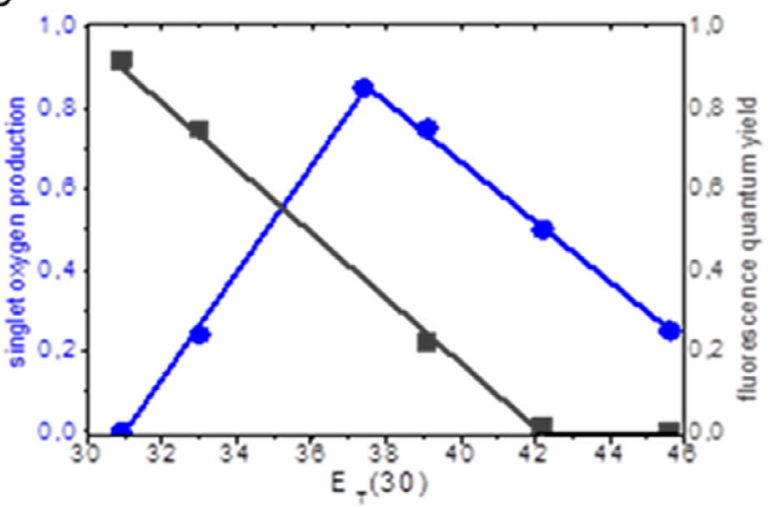

Figure 11. (A) Normalized absorption and emission spectra of 25 in different solvents: cyclohexane (black), toluene (green), $\mathrm{CHCl}_{3}$ (red), acetone (purple) and ACN (blue); inset: normalized emission spectra in acetone and ACN. (B) Energy diagram of the key electronic levels of 25, and corresponding orbitals involved in the key energy transfers. (C) Linear correlation between the singlet oxygen quantum yield $\left(\Phi_{\Delta}\right)$ and fluorescence quantum yield $\left(\Phi_{\mathrm{ff}}\right)$ of $\mathbf{2 5}$ in different solvents and the solvent Reichardt parameter, $E_{\mathrm{T}}(30)$.

group (compound 27, Fig. 11) led to a higher ${ }^{1} \mathrm{O}_{2}$ generation with respect to that exhibited by compound $25\left(\Phi_{\Delta}=0.85 \mathrm{vs}\right.$ 0.75 in $\mathrm{CHCl}_{3}$, Table 2). These two compounds (26 and 27) demonstrate that ${ }^{1} \mathrm{O}_{2}$ generation can be also modulated by introducing either electron-donating or electron-withdrawing groups in the structural design of the BODIPY dimer.

Finally, with the aim of developing PSs with redshifted absorption bands in the visible-near-infrared spectral region, $\pi$ conjugated systems were directly grafted at the BODIPY units. However, extending the $\pi$-conjugation by linking phenylethynyls or thienyls (see 28 and 29 in Fig. 10) at positions 2/6 and 6' of
Table 2. Photophysical properties of orthogonal BODIPY dimers in airequilibrated $\mathrm{CHCl}_{3}$.

\begin{tabular}{llllll}
\hline Dimer & $\lambda_{\text {abs }}(\mathrm{nm})$ & $\varepsilon_{\max }\left(\mathrm{M}^{-1} \mathrm{~cm}^{-1}\right)$ & $\lambda_{\text {flu }}(\mathrm{nm})$ & $\Phi_{\text {flu }}$ & $\Phi_{\Delta}$ \\
\hline $\mathbf{2 5}$ & 505 & 180000 & 527 & 0.22 & $0.75^{*}$ \\
$\mathbf{2 6}$ & 513 & 151000 & $532 / 675$ & 0.01 & $0.43^{*}$ \\
$\mathbf{2 7}$ & 510 & 81000 & 526 & 0.05 & $0.85^{*}$ \\
$\mathbf{2 8}$ & $550 / 585$ & 110000 & 615 & 0.24 & $0.17^{*}$ \\
$\mathbf{2 9}$ & 525 & 102000 & 627 & 0.17 & $0.12^{*}$ \\
$\mathbf{3 0}$ & 509 & 35000 & 685 & $0.56 \S$ & $0.11^{*}$ \\
& 658 & 58000 & & & $0.01 \dagger$ \\
$\mathbf{3 1}$ & 510 & 31000 & 613 & $0.22 \S$ & $0.20^{*}$ \\
& 589 & 45000 & & & $0.07 \dagger$ \\
$\mathbf{3 2}$ & 427 & 8400 & 573 & $0.19 \S$ & $0.53^{*}+\S$ \\
& 522 & 10700 & & & \\
& 544 & 12500 & & &
\end{tabular}

*Green excitation: 17 in $\mathrm{CHCl}_{3}$ used as the reference for quantifying $\Phi_{\Delta}$ (69). $†$ Red excitation: methylene blue in $\mathrm{CHCl}_{3}$ used as the reference for quantifying $\Phi_{\Delta} . \dagger \mathrm{UV}$-blue excitation, $\mathrm{PN}$ in $\mathrm{CHCl}_{3}$ used as the reference for quantifying $\Phi_{\Delta}$. $\S$ Independent from the excitation wavelength.

the BODIPY did not generate significant redshifts of the absorption bands, and modest $\Phi_{\Delta}(<0.20)$ were reached (Table 2$)$.

Therefore, molecular designs based on orthogonal BODIPY dimers with asymmetric $\pi$-substitution in positions 3 and/or 5 in only one of the BODIPY units were synthesized and studied (compounds $\mathbf{3 0}$ and $\mathbf{3 1}$ in Fig. 10). In these cases, the electronic delocalization induced the largest energy difference between the HOMO and HOMO-1, and the LUMO+1 and LUMO states, so that they cannot be anymore considered as nearly degenerated. Consequently, well-differentiated bands were recorded in their respective absorption spectra (Table 2), which is detrimental to ${ }^{1} \mathrm{O}_{2}$ generation. Note that, both compounds, 30 and $\mathbf{3 1}$, exhibit a modest ${ }^{1} \mathrm{O}_{2}$ production under green irradiation $\left(\Phi_{\Delta}=0.11\right.$ and 0.22 , Table 2) but only compound $\mathbf{3 1}$ shows just a little ${ }^{1} \mathrm{O}_{2}$ generation under red excitation $\left(\Phi_{\Delta}=0.07\right.$, Table 2$)$. Thus, removal of a single styryl group in $\mathbf{3 0}$, to generate $\mathbf{3 1}$, leads to a better balance between fluorescence efficiency and ${ }^{1} \mathrm{O}_{2}$ generation under irradiation with light of ca. $600 \mathrm{~nm}$ although they remain still low $\left(\Phi_{\text {flu }}=0.22, \Phi_{\Delta}=0.07 v s \Phi_{\text {flu }}=0.54, \Phi_{\Delta}=0.01\right.$ for compounds 31 and 30, respectively, Table 2). Accordingly, the development of advanced halogen-free PSs with high ${ }^{1} \mathrm{O}_{2}$ generation for PDT or with dual functionality $\left({ }^{1} \mathrm{O}_{2}\right.$ generation + fluorescence) for theragnostic applications (PDT + imaging) upon excitation in the therapeutic window seems to be limited in these type of orthogonal BODIPY-based PSs.

However, from in vitro studies conducted on HeLa cells, we found interesting results from selected dimers $(\mathbf{2 5}, 27$ and 31) as potential theragnostic biomaterials under green excitation since these dimers efficiently internalize into HeLa cells, exhibiting strong fluorescence and high phototoxicity under green light without any dark toxicity (Fig. 12A and B) (12). Particularly, after incubating the cells with $100 \mathrm{~nm} 25$ for 24 h, a full lethal effect is achieved for low green light irradiation doses $\left(5 \mathrm{~J} \mathrm{~cm}^{-2}\right)$. Interestingly, at lower dye concentration (less than $50 \mathrm{~nm}$ ), $<10 \%$ of cell survival was achieved. This result is attributed to the intrinsic high phototoxic power $\left(P P\right.$, defined as $\left.P P=\varepsilon \cdot \Phi_{\Delta}\right)$ of 25 ( $\mathrm{PP}>200.000 \mathrm{~cm}^{-1} \mathrm{M}^{-1}$ ), which is crucial to minimize side effects on potential patients since efficiency is reached without the necessity of using high PS doses and/or extended high-energy treatments (Fig. 12C). Moreover, the PP value of 25 is well balanced with its brightness (BT, defined as BT $=\varepsilon \cdot \Phi_{\text {flu }} ; \sim 4 \times$ 

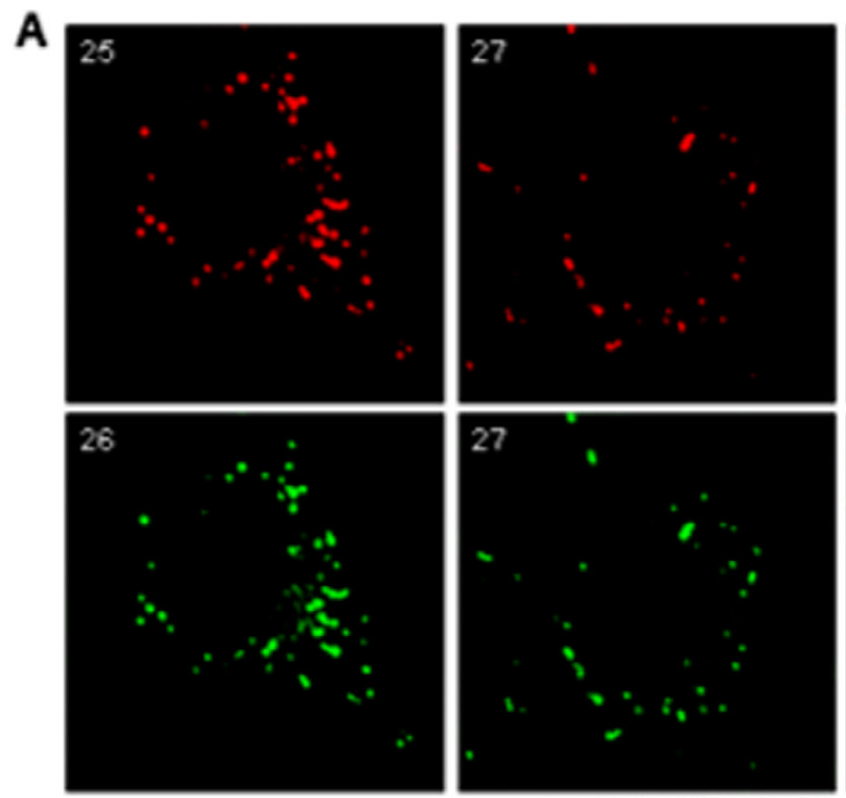

DARK TOXICITY

B

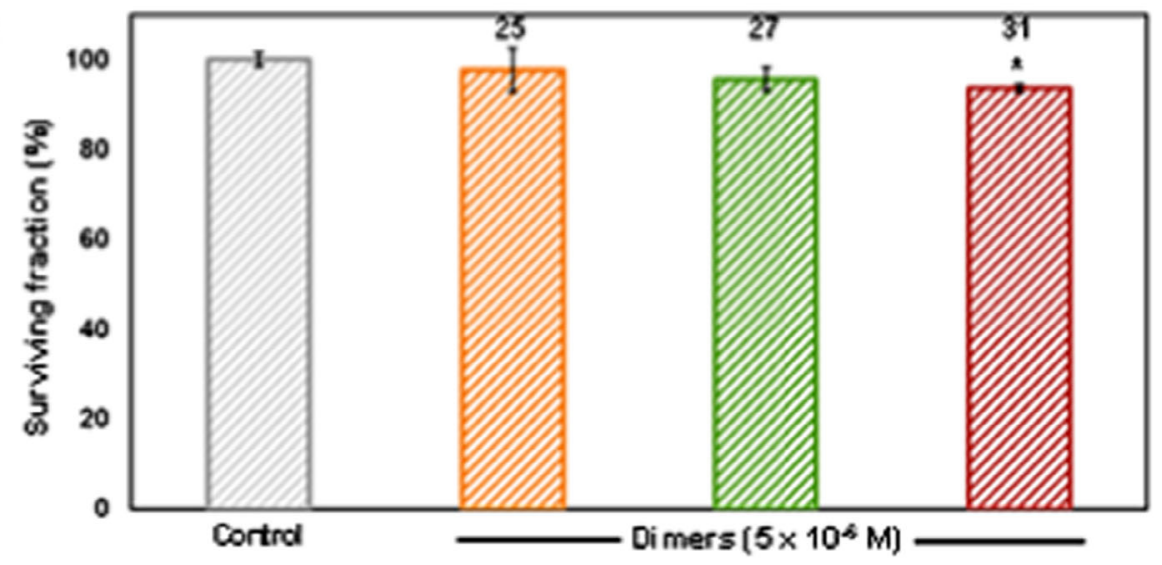

C

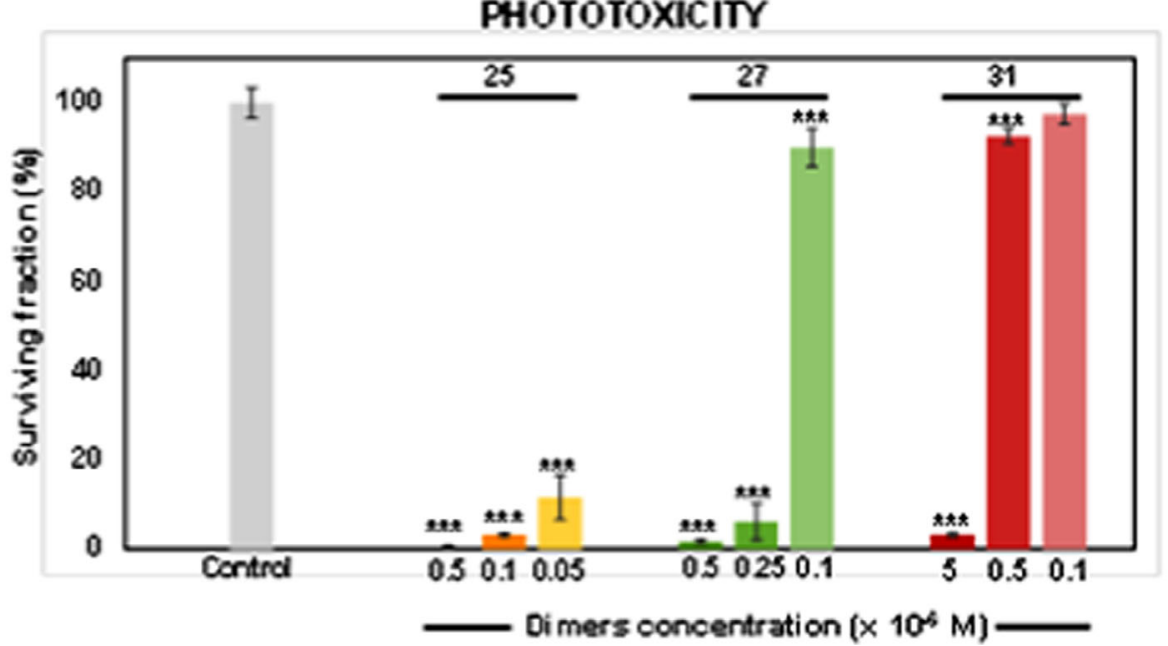

Figure 12. Theragnostic activity of $\mathbf{2 5}, \mathbf{2 7}$ and $\mathbf{3 1}$ on HeLa cells. (A) Confocal microscopy images of living HeLa cells incubated with $5 \times 10^{-6}{ }_{\mathrm{M}}$ dye for $24 \mathrm{~h}$. Images were obtained by using Vis laser irradiation $(488 \mathrm{~nm})$ and recording the emission at 587-673 and $497-552 \mathrm{~nm}$ for red and green images, respectively. Scale bar: $5 \mu \mathrm{m}$. (B) Surviving fractions of HeLa cells after 24 -h incubation with the corresponding dye $\left(5 \times 10^{-6} \mathrm{M}\right)$ in the absence of irradiation (dark toxicity). (C) Phototoxicity of HeLa cells after 24-h incubation with the corresponding dye at different concentrations, followed by $5 \mathrm{~J} \mathrm{~cm}^{-2}$ of green light irradiation. Dark toxicity and phototoxicity were determined by using MTT assay. Data correspond to mean $\pm \mathrm{SD}$ values from at least six different experiments. Statistically significant differences are labeled as $*(P<0.01), * *(P<0.005)$ and $* * *(P<0.001)$ for comparisons between groups using one-way ANOVA. 


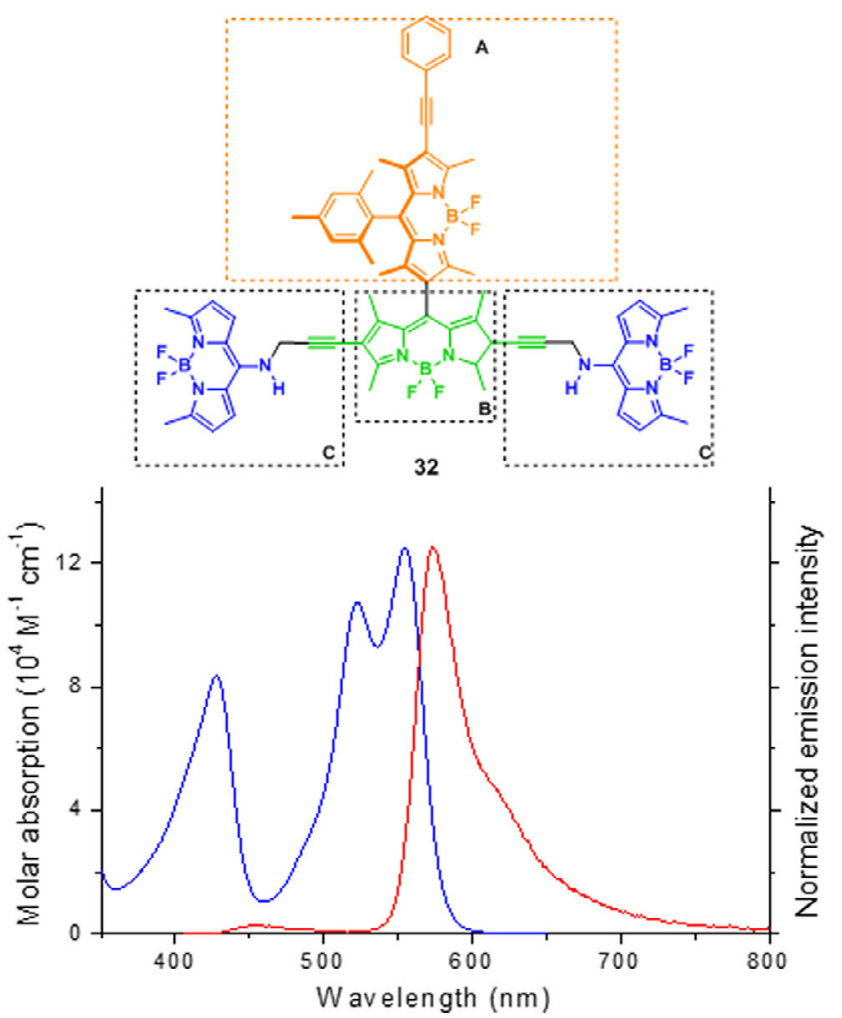

Figure 13. Molecular structure of 32 (key BODIPY moieties in different colors and key building-block fragments into dotted windows), as well as its absorption (blue) and normalized fluorescence (red) spectra (emission spectra obtained by exciting the "blue" chromophore at $400 \mathrm{~nm}$ ).

$10^{4} \mathrm{~cm}^{-1} \mathrm{M}^{-1}$ for 25) confirming the great potential of this dimeric dye as PDT + bioimaging theragnostic agent.

The gained information on the rational design of BODIPY dyes for potential PDT applications prompted us to develop a multichromophoric dye, based on four interconnected BODIPY chromophores, to achieve high PP and BT under white light irradiation (see 32 in Fig. 13). This is an interesting issue since photoactivation by white light allows topic daylight treatments of different skin disorders (acne, keratosis, skin tumors, etc.) produced by fungi, bacteria, etc (115-121).

The molecular design of compound $\mathbf{3 2}$ consists of a fluorescent orange-emitting BODIPY fluorophore (fragment $\mathrm{A}$ in Fig. 13) orthogonally linked to a dissimilar, green-absorbing BODIPY chromophore (fragment B in Fig. 13). For widening the absorption spectral window, two additional blue-absorbing 8aminoBODIPY chromophores (fragment $\mathrm{C}$ in Fig. 13) were tethered to central B through a methylene-ethynyl spacer to avoid electronic interaction between them (13).

As a result, the absorption spectrum of $\mathbf{3 2}$ is nearly the combination of the bands of all the involved individual chromophores (Fig. 13). Thus, three strong absorption bands $\left(\varepsilon_{\max }>8 \times 10^{4} \mathrm{M}^{-1} \mathrm{~cm}^{-1}\right)$ were recorded at 427 ("blue" 8-aminoBODIPY), 522 ("green" BODIPY) and $554 \mathrm{~nm}$ ("orange" $\pi$-extended BODIPY). Regardless of the excitation wavelength (i.e. $400 \mathrm{~nm}$ at the blue fragment or $500 \mathrm{~nm}$ at the green fragment), just a single fluorescence band was recorded at $573 \mathrm{~nm}$, corresponding to the long-wavelength-emitting orange fluorophore (A; Fig. 13).

Therefore, from a photophysical point of view, the multichromophoric BODIPY $\mathbf{3 2}$ acts as an efficient molecular cassette, harvesting light simultaneously over a wide spectral region and transferring the excitation energy from the blue donor moiety to the final acceptor BODIPY in the orthogonal meso fragment by successive and efficient EET (excitation energy transfer) steps. Indeed, irrespective of the excitation wavelength, a good balance between fluorescence emission $\left(\Phi_{\text {flu }}=0.19\right.$, Table 2) and ${ }^{1} \mathrm{O}_{2}$ generation $\left(\Phi_{\Delta}=0.53\right.$, Table 2$)$ was reached, showing its potential as PDT + bioimaging theragnostic agent, which was confirmed by in vitro studies on HeLa cells.

Thus, 32 exhibits similar phototoxicity on HeLa cells under blue, green and, what is more important, white light irradiation, which was quantified by applying a photonic normalization criteria (13). On the other hand, it was also demonstrated that $\mathbf{3 2}$ efficiently internalizes into HeLa cells, being preferably accumulated into lysosomes to endow them with a brilliant red fluorescence (Fig. 14A). Although the fluorescence efficiency of $\mathbf{3 2}$ drops down to $6 \%$ in culture media, likely attributed to the stabilization of the ICT state competing with the radiative emission from the singlet state (fluorescence), the fluorescence of compound 32 is high enough to allow recording of sharp bioimages (Fig. 14).

The selective accumulation of $\mathbf{3 2}$ into lysosomes was confirmed by a colocalization experiment with LysoTracker ${ }^{\circledR}$ Green (Fig. 14B). The yellow signal obtained by overlapping the corresponding red and green signals (Fig. 14C) proves that 32 accumulates preferably into the lysosomal compartment with a significant selectivity. The measured Manders' overlap coefficient (MOC) between 32 and LysoTracker ${ }^{\circledR}$ Green $(0.81 \pm 0.07)$ corroborates such a selectivity $(\mathrm{MOC}=1.0$ indicates perfect colocalization).

Moreover, a mitochondrial apoptotic pathway is involved in the phototoxicity activity of $\mathbf{3 2}$, as it was demonstrated by double-immunofluorescent labeling of cytochrome $\mathrm{C}$ and activated caspase-3, as well as by H-33258 DNA counterstaining (Fig. 14bottom) (13). In this regard, cells with morphological features of apoptosis showed cytochrome $\mathrm{C}$ release from mitochondria into cytosol ( $c f$. Fig. 14A and E), strong fluorescence signal from activated caspase-3 (cf. Fig. 14B and F) and condensed and/or fragmented nuclei (cf. Fig. 14C and G).

The outstanding physical, chemical and biological properties of 32, such as accessible synthesis, biocompatibility and biospecificity, low dark toxicity (note the absence of heavy atoms in the structure), synthetic accessibility, high fluorescence, high phototoxicity and, what is more important, white-light-triggered activity, make its structural design a valuable chemical platform for the development of future agents to be used in clinical theragnostic applications with the additional advantage of the open possibility for further fine-tuning of properties, allowed by the workable BODIPY chemistry.

\section{CONCLUSIONS}

This review shows the potential of novel BODIPY designs for PDT applications, due to the possibility of finely tuning key photophysical, chemical and biological properties by accessible BODIPY chemistry. Therefore, properly functionalized haloBODIPYs are shown to be valuable platforms for designing robust (photostable) ${ }^{1} \mathrm{O}_{2}$ PSs, able to endure high-density photoexcitation, as well as to develop functional nanoparticles endowed with PDT activity. On the other hand, certain bromoBODIPYs, BODIPY-involving Ir(III)-complexes and, particularly, heavy-atom-free orthogonal BODIPY dimers are shown as interesting platforms for 

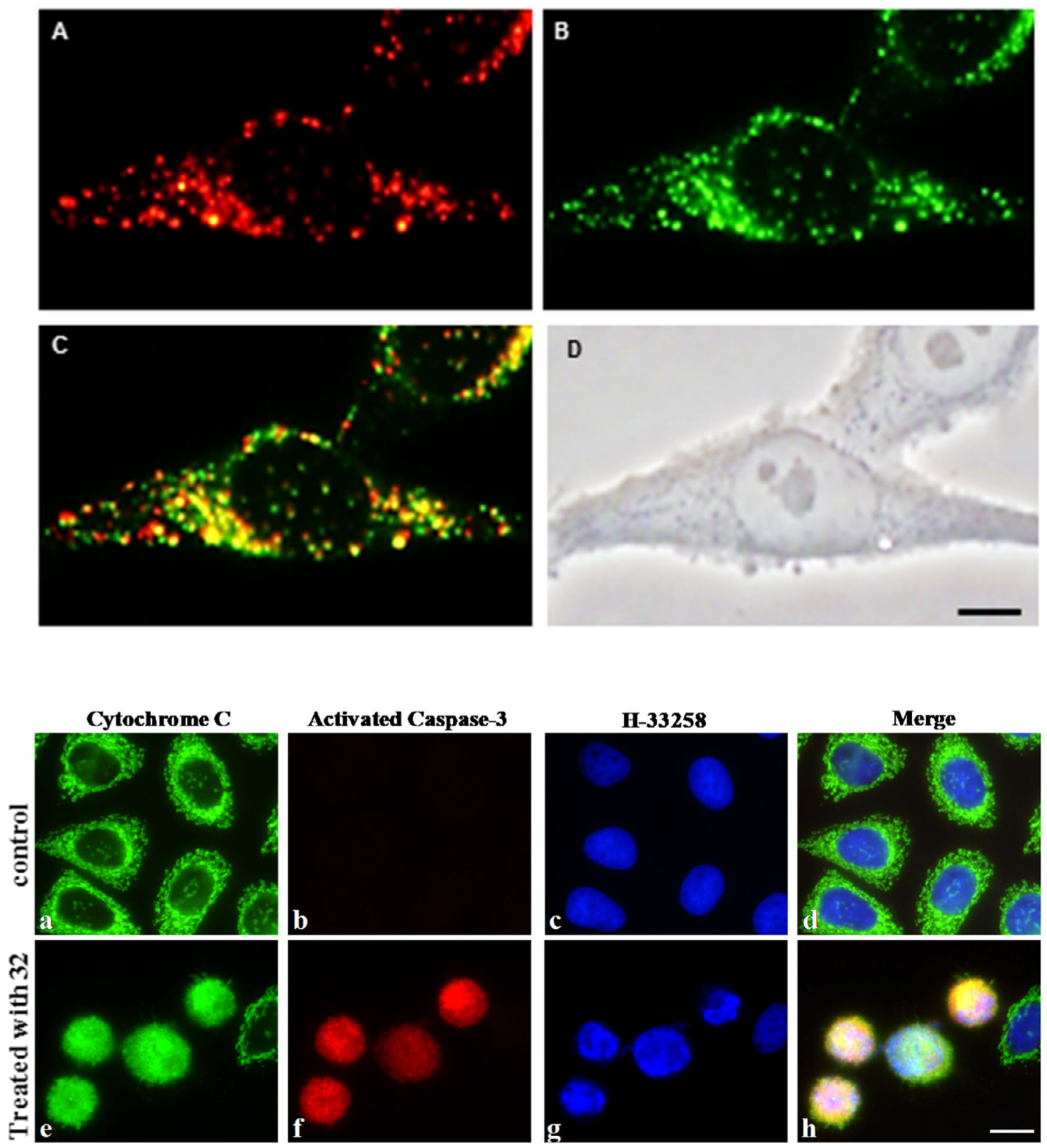

Figure 14. (TOP) Colocalization studies of $\mathbf{3 2}$ and LysoTracker Green in Hela. (A) Red fluorescence from 32 in HeLa cells. (B) Green fluorescence from LysoTracker ${ }^{\circledR}$ Green in HeLa cells, which is selective for acidic lysosome (or endosome). (C) Overlay of the red and green images. (D) Same field of cells visualized by phase-contrast microscopy. Scale bar: $5 \mu \mathrm{m}$. (BOTTOM) Apoptotic death of HeLa cells $24 \mathrm{~h}$ after photodynamic treatment with $32\left(10^{-5} \mathrm{M}+12.6 \mathrm{~J} \mathrm{~cm}^{-2}\right.$ green irradiation). Immunofluorescent localization of cytochrome C (green) and activated caspase-3 (red), DNA counterstaining with H-33258 (blue) and merged images in control (a-d) and photodynamically treated cells (e-h), respectively. Release of cytochrome C from mitochondria into the cytosol results in the activation of caspase-3. Scale bar: $5 \mu \mathrm{m}$.

developing future biomaterials for PDT + bioimaging theragnosis, due to their high enough fluorescence, making bioimaging possible, together with their ability for PDT. Particularly, orthogonal dimers show many advantages with respect to the rest of the BODIPY PDT PSs, such as high absorption coefficients and singlet oxygen production, offering high phototoxic action at low dye concentration (in the nm range) and low light irradiation doses. Moreover, they also show sufficient fluorescence to allow bioimaging (allowing them to be considered as suitable scaffolds for theragnostic applications), low cytotoxicity in the absence of light (mainly due to the absence of heavy atoms in the structure) and specific accumulation into lysosomes. However, the development of orthogonal BODIPY dimers working in the therapeutic spectral window $(>650 \mathrm{~nm})$ is limited. In this context, advanced BODIPY PSs with intense absorption bands in this region should be developed on the basis of different approaches, such as those including metal complexes in the molecular structure, or using halogenated BODIPY with specific substituents able to extend the electron $\pi$ delocalization of the acting BODIPY chromophore. Hence, there is still plenty of room for improvement, which drives us to continue working on the development of smarter PDT platforms based on BODIPY dyes able to be used in real clinical applications. 
"Somewhere, something incredible is waiting to be known.” Sharon Begley

Acknowledgements-The authors strongly acknowledge the contribution of their collaborators and coworkers cited in the listed references, as well. We thank the financial support obtained from MINECO (MAT2014-51937-C3-2-P and 3-P, MAT2017-83856-C3-2-P and 3-P, MAT2015-68837-REDT and CTQ2016-78454-C2-2-R), Comunidad de Madrid (S2013/MIT-2850) and Gobierno Vasco (IT912-16) of Spain. IMDEA Nanoscience Institute acknowledges support from the "Severo Ochoa" Programme for Centers of Excellence in R\&D (MINECO, Grant SEV-2016-0686).

\section{REFERENCES}

1. Nonell, S. and C. Flors (2016) Singlet oxygen. (Edited by S. Nonell and C. Flors). Royal Society of Chemistry, Cambridge.

2. Ogilby, P. R. (2010) Singlet oxygen: There is indeed something new under the sun. Chem. Soc. Rev. 39, 3181.

3. DeRosa, M. C. and R. J. Crutchley (2002) Photosensitized singlet oxygen and its applications. Coord. Chem. Rev. 233-234, $351-371$.

4. Deng, K., C. Li, S. Huang, B. Xing, D. Jin, Q. Zeng, Z. Hou and J. Lin (2017) Recent progress in near infrared light triggered photodynamic therapy. Small 13, 1-27.

5. Dichiara, M., O. Prezzavento, A. Marrazzo, V. Pittalà, L. Salerno, A. Rescifina and E. Amata (2017) Recent advances in drug discovery of phototherapeutic non-porphyrinic anticancer agents. Eur. J. Med. Chem. 142, 459-485.

6. Li, B., L. Lin, H. Lin and B. C. Wilson (2016) Photosensitized singlet oxygen generation and detection: Recent advances and future perspectives in cancer photodynamic therapy. J. Biophotonics $\mathbf{9}$, 1314-1325.

7. Abrahamse, H. and M. R. Hamblin (2016) New photosensitizers for photodynamic therapy. Biochem. J. 473, 347-364.

8. Zhao, J., W. Wu, J. Sun and S. Guo (2013) Triplet photosensitizers: from molecular design to applications. Chem. Soc. Rev. 42, 53235351.

9. Awuah, S. G. and Y. You (2012) Boron dipyrromethene (BODIPY)-based photosensitizers for photodynamic therapy. RSC Adv. 2, 11169.

10. Kamkaew, A., S. H. Lim, H. B. Lee, L. V. Kiew, L. Y. Chung and K. Burgess (2013) BODIPY dyes in photodynamic therapy. Chem. Soc. Rev. 42, 77-88.

11. Turksoy, A., D. Yildiz and E. U. Akkaya (2019) Photosensitization and controlled photosensitization with BODIPY dyes. Coord. Chem. Rev. 379, 47-64.

12. Li, X., S. Kolemen, J. Yoon and E. U. Akkaya (2017) Activatable photosensitizers: agents for selective photodynamic therapy. $A d v$. Funct. Mater. 27, 1604053.

13. Marfin, Y. S., A. V. Solomonov, A. S. Timin and E. V. Rumyantsev (2017) Recent advances of individual BODIPY and BODIPYbased functional materials in medical diagnostics and treatment. Curr. Med. Chem. 24, 2745.

14. Wilkinson, F., W. P. Helman and A. B. Ross (1993) Quantum yield for the photosensitized formation of the lowest electronically excited singlet state of molecular oxygen in solution. J. Phys. Chem. Ref. Data 22, 113-262.

15. Schweitzer, C. and R. Schmidt (2003) Physical mechanisms of generation and deactivation of singlet oxygen. Chem. Rev. 103, 16851758.

16. Zhou, Z., J. Song, L. Nie and X. Chen (2016) Reactive oxygen species generating systems meeting challenges of photodynamic cancer therapy. Chem. Soc. Rev. 45, 6597-6626.

17. Turro, N. J. (1991) Modern Molecular Photochemistry. University Science Books. page 117.

18. Braun, A. M. and E. Oliveros (1990) Applications of singlet oxygen reactions: mechanistic and kinetic investigations. Pure Appl. Chem. 62, 1467-1476.
19. Martinez, C. G., A. Neuner, C. Martí, S. Nonell, A. M. Braun and E. Oliveros (2003) Effect of the media on the quantum yield of singlet oxygen $\left(\mathrm{O}_{2}\left({ }^{1} \Delta_{\mathrm{g}}\right)\right)$ production by $9 \mathrm{H}$-fluoren-9-one: Solvents and solvent mixtures. Helv. Chim. Acta 86, 384-397.

20. Prieto-Montero, R., R. Sola-Llano, R. Montero, A. Longarte, T. Arbeloa, I. López-Arbeloa, V. Martínez-Martínez and S. Lacombe (2019) Methylthio BODIPY as a standard triplet photosensitizer for singlet oxygen production: A photophysical study. Phys. Chem. Chem. Phys. 2, 20403-20414.

21. Huang, Z. (2005) A review of progress in clinical photodynamic therapy. Technol. Cancer Res. Treat. 4, 283-293.

22. Kataoka, H., H. Nishie, N. Hayashi, M. Tanaka, A. Nomoto, S. Yano and T. Joh (2017) New photodynamic therapy with next-generation photosensitizers. Ann. Transl. Med. 5, 1-7.

23. van Straten, D., V. Mashayekhi, H. S. de Bruijn, S. Oliveira and D. J. Robinson (2017) Oncologic photodynamic therapy: Basic principles, current clinical status and future directions. Cancers 9, 1-54.

24. Ormond, A. B. and H. S. Freeman (2013) Dye Sensitizers for Photodynamic Therapy. Materials 6, 817-840.

25. Yoon, I., J. Z. Li and Y. K. Shim (2013) Advance in photosensitizers and light delivery for photodynamic therapy. Clin. Endosc. 46, 7-23.

26. Yang, X., P. Palasuberniam, D. Kraus and B. Chen (2015) Aminolevulinic acid-based tumor detection and therapy: Molecular mechanisms and strategies for enhancement. Int. J. Mol. Sci. 16, 25865-25880.

27. Zhu, W., Y. H. Gao, C. H. Song, Z. Bin Lu, T. Namulinda, Y. P. Han, Y. J. Yan, L. X. Wang and Z. L. Chen (2017) Synthesis and evaluation of new 5-aminolevulinic acid derivatives as prodrugs of protoporphyrin for photodynamic therapy. Photochem. Photobiol. Sci. 16, 1623-1630.

28. Kawczyk-Krupka, A., A. M. Bugaj, W. Latos, K. Zaremba, K. Wawrzyniec, M. Kucharzewski and A. Sieroń (2016) Photodynamic therapy in colorectal cancer treatment-The state of the art in preclinical research. Photodiagnosis Photodyn. Ther. 13, 158-174.

29. Krzykawska-Serda, M., J. M. Da̧browski, L. G. Arnaut, M. Szczygieł, K. Urbańska, G. Stochel and M. Elas (2014) The role of strong hypoxia in tumors after treatment in the outcome of bacteriochlorin-based photodynamic therapy. Free Radic. Biol. Med. 73, 239-251.

30. Rocha, L. B., L. C. Gomes-Da-Silva, J. M. Dąbrowski and L. G. Arnaut (2015) Elimination of primary tumours and control of metastasis with rationally designed bacteriochlorin photodynamic therapy regimens. Eur. J. Cancer 51, 1822-1830.

31. O'Connor, A. E., W. M. Gallagher and A. T. Byrne (2009) Porphyrin and nonporphyrin photosensitizers in oncology: Preclinical and clinical advances in photodynamic therapy. Photochem. Photobiol. 85, 1053-1074.

32. Dạbrowski, J. M. and L. G. Arnaut (2015) Photodynamic therapy (PDT) of cancer: From local to systemic treatment. Photochem. Photobiol. Sci. 14, 1765-1780.

33. McKenzie, L. K., I. V. Sazanovich, E. Baggaley, M. Bonneau, V. Guerchais, J. A. G. Williams, J. A. Weinstein and H. E. Bryant (2017) Metal complexes for two-photon photodynamic therapy: A cyclometallated Iridium complex induces two-photon photosensitization of cancer cells under near-IR light. Chem. Eur. J. 23, 234238.

34. Huang, Y.-Y., S. K. Sharma, R. Yin, T. Agrawal, Long Y. Chiang and M. R. Hamblin (2014) Functionalized fullerenes in photodynamic therapy. J. Biomed. Nanotechnol. 10, 1918-1936.

35. Loudet, A. and K. Burgess (2007) BODIPY dyes and their derivatives: Syntheses and spectroscopic properties. Chem. Rev. 107, 4891-4932.

36. Ulrich, G., R. Ziessel and A. Harriman (2008) The chemistry of fluorescent bodipy dyes: Versatility unsurpassed. Angew. Chem. Int. Ed. 47, 1184-1201.

37. Benniston, A. C. and G. Copley (2009) Lighting the way ahead with boron dipyrromethene (Bodipy) dyes. Phys. Chem. Chem. Phys. 11, 4124-4131.

38. Benstead, M., G. H. Mehl and R. W. Boyle (2011) 4,4'-Difluoro-4bora-3a,4a-diaza-s-indacenes (BODIPYs) as components of novel light active materials. Tetrahedron 67, 3573-3601.

39. Boens, N., V. Leen and W. Dehaen (2012) Fluorescent indicators based on BODIPY. Chem. Soc. Rev. 41, 1130-1172. 
40. Lu, H., J. Mack, Y. Yang and Z. Shen (2014) Structural modification strategies for the rational design of red/NIR region BODIPYs. Chem. Soc. Rev. 43, 4778-4823.

41. Kowada, T., H. Maeda and K. Kikuchi (2015) BODIPY-based probes for the fluorescence imaging of biomolecules in living cells. Chem. Soc. Rev. 44, 4953-4972.

42. Bañuelos, J. (2016) BODIPY Dye, the most versatile fluorophore ever? Chem. Rec. 16, 335-348.

43. Kolemen, S. and E. U. Akkaya (2018) Reaction-based BODIPY probes for selective bio-imaging. Coord. Chem. Rev. 354, 121-134.

44. Boens, N., B. Verbelen and W. Dehaen (2015) Postfunctionalization of the BODIPY core: Synthesis and spectroscopy. Eur. J. Org. Chem. 2015, 6577-6595.

45. Agazzi, M. L., M. B. Ballatore, A. M. Durantini, E. N. Durantini and A. C. Tomé (2019) BODIPYs in antitumoral and antimicrobial photodynamic therapy: An integrating review. J. Photoch. Photobiol. C 40, 21-48.

46. Lim, S. H., C. Thivierge, P. Nowak-Sliwinska, J. Han, H. Van Den Bergh, G. Wagnières, K. Burgess and H. B. Lee (2010) In vitro and in vivo photocytotoxicity of boron dipyrromethene derivatives for photodynamic therapy. J. Med. Chem. 53, 2865-2874.

47. Yogo, T., Y. Urano, Y. Ishitsuka, F. Maniwa and T. Nagano (2005) Highly efficient and photostable photosensitizer based on BODIPY chromophore. J. Am. Chem. Soc. 127, 12162-12163.

48. Wang, S., R. Gao, F. Zhou and M. Selke (2004) Nanomaterials and singlet oxygen photosensitizers: Potential applications in photodynamic therapy. J. Mater. Chem. 14, 487-493.

49. Cotí, K. K., M. E. Belowich, M. Liong, M. W. Ambrogio, Y. A. Lau, H. A. Khatib, J. I. Zink, N. M. Khashab and J. F. Stoddart (2009) Mechanised nanoparticles for drug delivery. Nanoscale 1, $16-39$.

50. Wang, Y., Q. Zhao, N. Han, L. Bai, J. Li, J. Liu, E. Che, L. Hu, Q. Zhang, T. Jiang and S. Wang (2015) Mesoporous silica nanoparticles in drug delivery and biomedical applications. Nanomed. Nanotechnol. 11, 313-327.

51. Mamaeva, V., C. Sahlgren and M. Lindén (2013) Mesoporous silica nanoparticles in medicine-Recent advances. Adv. Drug Deliv. Rev. 65, 689-702.

52. Zhou, L., W. Wang, S. H. Wei, Y. Y. Feng, J. H. Zhou, J. H. Liu and J. Shen (2011) Encapsulation of hydrophobic anticancer drug in nano-scale porous ceramic materials for photodynamic therapy. J. Porous Mater. 18, 517-522.

53. Wang, H., R. Han, L. Yang, J. Shi, Z. Liu, Y. Hu, Y. Wang, S. Liu and Y. Gan (2016) Design and synthesis of core-shell-shell upconversion nanoparticles for NIR-induced drug release, photodynamic therapy, and cell imaging. ACS Appl. Mater. Interfaces 8 , 4416-4423.

54. Ozdemir, T., Y.-C. Lu, S. Kolemen, E. Tanriverdi-Ecik and E. U. Akkaya (2017) Generation of singlet oxygen by persistent luminescent nanoparticle-photosensitizer conjugates: A proof of principle for photodynamic therapy without light. ChemPhotoChem 1, 183187

55. Kilic, B., N. Yesilgul, V. Polat, Z. Gercek and E. U. Akkaya (2015) Bodipy-based photosensitizers with long alkyl tails at the meso position: Efficient singlet oxygen generation in CremophorEL micelles. Tetrahedron Lett. 57, 1317-1320.

56. Turan, I. S., D. Yildiz, A. Turksoy, G. Gunaydin and E. U. Akkaya (2016) A bifunctional photosensitizer for enhanced fractional photodynamic therapy: Singlet oxygen generation in the presence and absence of light. Angew. Chem. Int. Ed. 55, 2875-2878.

57. Erbas-Cakmak, S., F. P. Cakmak, S. D. Topel, T. B. Uyar and E. U. Akkaya (2015) Selective photosensitization through an and logic response: Optimization of the $\mathrm{pH}$ and glutathione response of activatable photosensitizers. Chem. Commun. 51, 12258-12261.

58. Turan, I. S., F. P. Cakmak, D. C. Yildirim, R. Cetin-Atalay and E. U. Akkaya (2014) Near-IR absorbing BODIPY derivatives as glutathione-activated photosensitizers for selective photodynamic action. Chem. Eur. J. 20, 16088-16092.

59. Erbas-Cakmak, S. and E. U. Akkaya (2014) Toward singlet oxygen delivery at a measured rate: A self-reporting photosensitizer. Org. Lett. 16, 2946-2949.

60. Erbas, S., A. Gorgulu, M. Kocakusakogullari and E. U. Akkaya (2009) Non-covalent functionalized SWNTs as delivery agents for novel Bodipy-based potential PDT sensitizers. Chem. Commun. 33 4956-4958.

61. Ozlem, S. and E. U. Akkaya (2009) Thinking outside the silicon box: Molecular and logic as an additional layer of selectivity in singlet oxygen generation for photodynamic therapy. J. Am. Chem. Soc. 131, 48-49.

62. Kue, C. S., A. Kamkaew, S. H. Voon, L. V. Kiew, L. Y. Chung, K. Burgess and H. B. Lee (2016) Tropomyosin receptor kinase C targeted delivery of a peptidomimetic ligand-Photosensitizer conjugate induces antitumor immune responses following photodynamic therapy. Sci. Rep. 6, 1-17.

63. Kamkaew, A. and K. Burgess (2013) Double-targeting using a TrkC ligand conjugated to dipyrrometheneboron difluoride (BODIPY) based photodynamic therapy (PDT) agent. J. Med. Chem. 56, 7608-7614.

64. Wu, W., Y. Geng, W. Fan, Z. Li, L. Zhan, X. Wu, J. Zheng, J. Zhao and M. Wu (2014) BODIPY-based photosensitizers with intense visible light harvesting ability and high ${ }^{1} \mathrm{O}_{2}$ quantum yield in aqueous solution. $R S C A d v$. 4, 51349-51352.

65. Ge, Y. and D. F. O'Shea (2016) Azadipyrromethenes: From traditional dye chemistry to leading edge applications. Chem. Soc. Rev 45, 3846-3864.

66. Zhang, X. F. and X. Yang (2013) Singlet oxygen generation and triplet excited-state spectra of brominated BODIPY. J. Phys. Chem. B 117, 5533-5539.

67. He, H., P. C. Lo, S. L. Yeung, W. P. Fong and D. K. P. Ng (2011) Synthesis and in vitro photodynamic activities of pegylated distyryl boron dipyrromethene derivatives. J. Med. Chem. 54, 3097-3102.

68. Banfi, S., E. Caruso, S. Zaza, M. Mancini, M. B. Gariboldi and E. Monti (2012) Synthesis and photodynamic activity of a panel of BODIPY dyes. J. Photochem. Photobiol. B. 114, 52-60.

69. Gibbs, J. H., Z. Zhou, D. Kessel, F. R. Fronczek, S. Pakhomova and M. G. H. Vicente (2015) Synthesis, spectroscopic, and in vitro investigations of 2,6-diiodo-BODIPYs with PDT and bioimaging applications. J. Photochem. Photobiol. B Biol. 145, 35-47.

70. Zou, J., Z. Yin, K. Ding, Q. Tang, J. Li, W. Si, J. Shao, Q. Zhang, W. Huang and X. Dong (2017) BODIPY derivatives for photodynamic therapy: influence of configuration versus heavy atom effect. ACS Appl. Mater. Interfaces 9, 32475-32481.

71. Gorbe, M., A. Barba-Bon, C. de la Torre, S. Gil, A. M. Costero, F. Sancenón, J. R. Murguía and R. Martínez-Máñez (2015) Synthesis and in vitro evaluation of a photosensitizer-BODIPY derivative for potential photodynamic therapy applications. Chem. Asian J. 10, 2121-2125.

72. Wang, C. and Y. Qian (2019) A novel BODIPY-based photosensitizer with $\mathrm{pH}$-active singlet oxygen generation for photodynamic therapy in lysosomes. Org. Biomol. Chem. 17, 8001-8007.

73. Durantini, A. M., L. E. Greene, R. Lincoln, S. R. Martínez and G. Cosa (2016) Reactive oxygen species mediated activation of a dormant singlet oxygen photosensitizer: From autocatalytic singlet oxygen amplification to chemicontrolled photodynamic therapy. J. Am Chem. Soc. 138, 1215-1225.

74. Ortiz, M. J., A. R. Agarrabeitia, G. Duran-Sampedro, J. Bañuelos Prieto, T. A. Lopez, W. A. Massad, H. A. Montejano, N. A. García and I. Lopez-Arbeloa (2012) Synthesis and functionalization of new polyhalogenated BODIPY dyes. Study of their photophysical properties and singlet oxygen generation. Tetrahedron 68, 1153-1162.

75. Sánchez-Arroyo, A. J., E. Palao, A. R. Agarrabeitia, M. J. Ortiz and D. García-Fresnadillo (2017) Towards improved halogenated BODIPY photosensitizers: clues on structural designs and heavy atom substitution patterns. Phys. Chem. Chem. Phys. 19, 69-72.

76. Epelde-Elezcano, N., V. Martínez-Martínez, E. Peña-Cabrera, C. F. A. Gómez-Durán, I. López-Arbeloa and S. Lacombe (2016) Modulation of singlet oxygen generation in halogenated BODIPY dyes by substitution at their meso position: towards a solvent-independent standard in the Vis region. RSC Adv. 6, 41991-41998.

77. Epelde-Elezcano, N., R. Prieto-Montero, V. Martínez-Martínez, M J. Ortiz, A. Prieto-Castañeda, E. Peña-Cabrera, J. L. BelmonteVázquez, I. López-Arbeloa, R. Brown and S. Lacombe (2017) Adapting BODIPYs to singlet oxygen production on silica nanoparticles. Phys. Chem. Chem. Phys. 19, 13746-13755.

78. Esnal, I., I. Valois-Escamilla, C. F. A. Gómez-Durán, A. UríasBenavides, M. L. Betancourt-Mendiola, I. Lõpez-Arbeloa, J. Bañuelos, I. García-Moreno, A. Costela and E. Peña-Cabrera (2013) 
Blue-to-orange color-tunable laser emission from tailored borondipyrromethene dyes. ChemPhysChem 14, 4134-4142.

79. Esnal, I., A. Urías-Benavides, C. F. A. Gõmez-Durán, C. A. OsorioMartínez, I. García-Moreno, A. Costela, J. Bañuelos, N. Epelde, I. López Arbeloa, R. Hu, B. Z. Tang and E. Peña-Cabrera (2013) Reaction of amines with 8-methylthioBODIPY: Dramatic optical and laser response to amine substitution. Chem. Asian J. 8, 2691-2700.

80. Osorio-Martínez, C., A. Urías-Benavides, A. Gómez-Durán, J. Bañuelos, I. Esnal, I. López-Arbeloa and E. Peña-Cabrera (2012) 8AminoBODIPYs: Cyanines or hemicyanines? The effect of the coplanarity of the amino group on their optical properties. J. Org. Chem. 77, 5434-5438.

81. Kabanov, V., D. J. Press, R. P. S. Huynh, G. K. H. Shimizu and B. Heyne (2018) Assessment of encapsulated dyes' distribution in silica nanoparticles and their ability to release useful singlet oxygen. Chem. Commun. 54, 6320-6323.

82. Black, F. A., A. Jacquart, G. Toupalas, S. Alves, A. Proust, I. P. Clark, E. A. Gibson and G. Izzet (2018) Rapid photoinduced charge injection into covalent polyoxometalate-bodipy conjugates. Chem. Sci. 9, 5578-5584.

83. Grazon, C., Y. Si, J. P. Placial, J. Rieger, R. Méallet-Renault and G. Clavier (2019) Core-shell polymeric nanoparticles comprising BODIPY and fluorescein as ultra-bright ratiometric fluorescent $\mathrm{pH}$ sensors. Photochem. Photobiol. Sci. 18, 1156-1165.

84. Valtchev, V. and L. Tosheva (2013) Porous nanosized particles: Preparation, properties, and applications. Chem. Rev. 113, 6734-60.

85. Lee, J. E., N. Lee, T. Kim, J. Kim and T. Hyeon (2011) Multifunctional mesoporous silica nanocomposite nanoparticles for theranostic applications. Acc. Chem. Res. 44, 893-902.

86. Malvindi, M. A., V. Brunetti, G. Vecchio, A. Galeone, R. Cingolani and P. P. Pompa (2012) $\mathrm{SiO}_{2}$ nanoparticles biocompatibility and their potential for gene delivery and silencing. Nanoscale $\mathbf{4}$, 486.

87. Djurovich, P. I., D. Murphy, M. E. Thompson, B. Hernandez, R. Gao, P. L. Hunt and M. Selke (2007) Cyclometalated Iridium and Platinum complexes as singlet oxygen photosensitizers: Quantum yields, quenching rates and correlation with electronic structures. Dalton Trans. 34, 3763-3770.

88. Rachford, A. A., R. Ziessel, T. Bura, P. Retailleau and F. N. Castellano (2010) Boron dipyrromethene (Bodipy) phosphorescence revealed in $\left[\operatorname{Ir}(\mathrm{ppy})_{2}(\mathrm{bpy}-\mathrm{C} \equiv \mathrm{C}-\mathrm{Bodipy})\right]^{+}$. Inorg. Chem. 49, 37303736.

89. Galletta, M., S. Campagna, M. Quesada, G. Ulrich and R. Ziessel (2005) The elusive phosphorescence of pyrromethene- $\mathrm{BF}_{2}$ dyes revealed in new multicomponent species containing $\mathrm{Ru}(\mathrm{II})$-terpyridine subunits. Chem. Commun. 33, 4222-4224.

90. Sun, H., H. Guo, W. Wu, X. Liu and J. Zhao (2011) Coumarin phosphorescence observed with $\mathrm{N}^{\wedge} \mathrm{N}$ Pt(II) bisacetylide complex and its applications for luminescent oxygen sensing and triplet-triplet-annihilation based upconversion. Dalton Trans. 40, 7834-7841.

91. Zhao, J., K. Xu, W. Yang, Z. Wang and F. Zhong (2015) The triplet excited state of Bodipy: Formation, modulation and application. Chem. Soc. Rev. 44, 8904-8939.

92. Sun, J., F. Zhong, X. Yi and J. Zhao (2013) Efficient enhancement of the visible-light absorption of cyclometalated $\operatorname{Ir}(\mathrm{III})$ complexes triplet photosensitizers with bodipy and applications in photooxidation and triplet-triplet annihilation upconversion. Inorg. Chem. 52, 6299-6310.

93. Palao, E., R. Sola-Llano, A. Tabero, H. Manzano, A. R. Agarrabeitia, A. Villanueva, I. López-Arbeloa, V. Martínez-Martínez and M. J. Ortiz (2017) AcetylacetonateBODIPY-biscyclometalated Iridium (III) complexes: Effective strategy towards smarter fluorescent photosensitizer agents. Chem. Eur. J. 23, 10139-10147.

94. Ventura, B., G. Marconi, M. Bröring, R. Krüger and L. Flamigni (2009) $\mathrm{Bis}\left(\mathrm{BF}_{2}\right)-2,2^{\prime}$-bidipyrrins, a class of BODIPY dyes with new spectroscopic and photophysical properties. New J. Chem. 33, 428-438.

95. Bröring, M., R. Krüger, S. Link, C. Kleeberg, S. Köhler, X. Xie, B. Ventura and L. Flamigni (2008) Bis $\left(\mathrm{BF}_{2}\right)-2,2$ '-bidipyrrins (BisBODIPYs): highly fluorescent BODIPY dimers with large stokes shifts. Chem. Eur. J. 14, 2976-83.

96. Duman, S., Y. Cakmak, S. Kolemen, E. U. Akkaya and Y. Dede (2012) Heavy atom free singlet oxygen generation: Doubly substituted configurations dominate $\mathrm{S}_{1}$ states of bis-BODIPYs. J. Org. Chem. 77, 4516-4527.
97. Topel, S. D., G. T. Cin and E. U. Akkaya (2014) Near IR excitation of heavy atom free Bodipy photosensitizers through the intermediacy of upconverting nanoparticles. Chem. Commun. 50, 88968899.

98. Pang, W., X.-F. Zhang, J. Zhou, C. Yu, E. Hao and L. Jiao (2012) Modulating the singlet oxygen generation property of meso- $\beta$ directly linked BODIPY dimers. Chem. Commun. 48, 5437-5439.

99. Wu, W., X. Cui and J. Zhao (2013) Hetero Bodipy-dimers as heavy atom-free triplet photosensitizers showing a long-lived triplet excited state for triplet-triplet annihilation upconversion. Chem. Commun. 49, 9009-9011.

100. Kolemen, S., M. Işık, G. M. Kim, D. Kim, H. Geng, M. Buyuktemiz, T. Karatas, X.-F. Zhang, Y. Dede, J. Yoon and E. U. Akkaya (2015) Intracellular modulation of excited-state dynamics in a chromophore dyad: Differential enhancement of photocytotoxicity targeting cancer cells. Angew. Chem. Int. Ed. 54, 5340-5344.

101. Zhang, X.-F., X. Yang and B. Xu (2017) PET-based bisBODIPY photosensitizers for highly efficient excited triplet state and singlet oxygen generation: tuning photosensitizing ability by dihedral angles. Phys. Chem. Chem. Phys. 19, 24792-24804.

102. Epelde-Elezcano, N., E. Palao, H. Manzano, A. Prieto-Castañeda, A. R. Agarrabeitia, A. Tabero, A. Villanueva, S. de la Moya, Í. López-Arbeloa, V. Martínez-Martínez and M. J. Ortiz (2017) Rational design of advanced photosensitizers based on orthogonal BODIPY dimers to finely modulate singlet oxygen generation. Chem. Eur. J. 23, 4837-4848.

103. Durán-Sampedro, G., N. Epelde-Elezcano, V. Martínez-Martínez, I. Esnal, J. Bañuelos, I. García-Moreno, A. R. Agarrabeitia, S. de la Moya, A. Tabero, A. Lazaro-Carrillo, A. Villanueva, M. J. Ortiz and I. López-Arbeloa (2017) A versatile fluorescent molecular probe endowed with singlet oxygen generation under white-light photosensitization. Dyes Pigm. 142, 77-87.

104. Whited, M. T., N. M. Patel, S. T. Roberts, K. Allen, P. I. Djurovich, S. E. Bradforth and M. E. Thompson (2012) Symmetry-breaking intramolecular charge transfer in the excited state of mesolinked BODIPY dyads. Chem. Commun. 48, 284-286.

105. Cakmak, Y., S. Kolemen, S. Duman, Y. Dede, Y. Dolen, B. Kilic, Z. Kostereli, L. T. Yildirim, A. L. Dogan, D. Guc and E. U. Akkaya (2011) Designing excited states: Theory-guided access to efficient photosensitizers for photodynamic action. Angew. Chem. Int. Ed. 50, 11937-11941.

106. Harriman, A., L. J. Mallon, G. Ulrich and R. Ziessel (2007) Rapid intersystem crossing in closely-spaced but orthogonal molecular dyads. ChemPhysChem 8, 1207-1214.

107. Benniston, A. C., S. Clift, J. Hagon, H. Lemmetyinen, N. V. Tkachenko, W. Clegg and R. W. Harrington (2012) Effect on charge transfer and charge recombination by insertion of a naphthalenebased bridge in molecular dyads based on borondipyrromethene (Bodipy). ChemPhysChem 13, 3672-3681.

108. Bandi, V., H. B. Gobeze, V. Lakshmi, M. Ravikanth and F. D'Souza (2015) Vectorial charge separation and selective tripletstate formation during charge recombination in a pyrrolyl-bridged BODIPY-fullerene dyad. J. Phys. Chem. C 119, 8095-8102.

109. Dance, Z. E. X., S. M. Mickley, T. M. Wilson, A. B. Ricks, A. M. Scott, M. A. Ratner and M. R. Wasielewski (2008) Intersystem crossing mediated by photoinduced intramolecular charge transfer: julolidine-anthracene molecules with perpendicular $\pi$ systems. $J$. Phys. Chem. A 112, 4194-4201.

110. Montero, R., V. Martínez-Martínez, A. Longarte, N. Epelde-Elezcano, E. Palao, I. Lamas, H. Manzano, A. R. Agarrabeitia, I. López Arbeloa, M. J. Ortiz and I. Garcia-Moreno (2018) Singlet fission mediated photophysics of BODIPY dimers. J. Phys. Chem. Lett. 9, 641-646.

111. Smith, J. M. (2010) Singlet fission. Chem. Rev. 110, 6891-6936.

112. Smith, M. B. and J. Michl (2013) Recent advances in singlet fission. Annu. Rev. Phys. Chem. 64, 361-386.

113. Scholes, G. D. (2015) Correlated pair states formed by singlet fission and exciton-exciton annihilation. J. Phys. Chem. A 119, 12699-12705.

114. Piland, G. B., J. J. Burdett, R. J. Dillon and C. J. Bardeen (2014) Singlet fission: From coherences to kinetics. J. Phys. Chem. Lett. 5, 2312-2319. 
115. Beyer, W., R. Waidelich, R. Knüchel, H. Stepp, R. Baumgartner and A. Hofstetter (2002) Technical concepts for white light photodynamic. Med. Laser Appl. 17, 37-40.

116. Dolmans, D. E. J. G. J., D. Fukumura and R. K. Jain (2003) Photodynamic therapy for cancer. Nat. Rev. Cancer 3, 380-387.

117. Tian, Z., W. Wu, W. Wan and A. D. Q. Li (2009) Single-chromophore-based photoswitchable nanoparticles enable dual-alternating-color fluorescence for unambiguous live cell imaging. J. Am. Chem. Soc. 131, 4245-4252.

118. Zhao, Q., J. Li, X. Zhang, Z. Li and Y. Tang (2016) Cationic oligo (thiophene ethynylene) with broad-spectrum and high antibacterial efficiency under white light and specific biocidal activity against $S$. aureus in dark. ACS Appl. Mater. Interfaces 8, 1019-1024.
119. Serebrovskaya, E. O., E. F. Edelweiss, O. A. Stremovskiy, K. A. Lukyanov, D. M. Chudakov and S. M. Deyev (2009) Targeting cancer cells by using an antireceptor antibody-photosensitizer fusion protein. Proc. Natl. Acad. Sci. USA 106, 9221-9225.

120. Zhang, C. J., Q. Hu, G. Feng, R. Zhang, Y. Yuan, X. Lu and B. Liu (2015) Image-guided combination chemotherapy and photodynamic therapy using a mitochondria-targeted molecular probe with aggregation-induced emission characteristics. Chem. Sci. 6, 4580-4586.

121. Francés-Soriano, L., M. A. Zakharko, M. González-Béjar, P. A. Panchenko, V. Herranz-Pérez, D. A. Pritmov, M. A. Grin, A. F. Mironov, J. M. García-Verdugo, O. A. Fedorova and J. Pérez-Prieto (2018) Nanohybrid for photodynamic therapy and fluorescence imaging tracking without therapy. Chem. Mater. 30, 3677-3682. 